\title{
A continuum of totally incomparable hereditarily indecomposable Banach spaces
}

\author{
by \\ I. Gasparis (Stillwater, OK, and Herakleion)
}

\begin{abstract}
A family is constructed of cardinality equal to the continuum, whose members are totally incomparable hereditarily indecomposable Banach spaces.
\end{abstract}

1. Introduction. All Banach spaces considered in this paper are real, infinite-dimensional. By a subspace of a Banach space we shall mean an infinite-dimensional, closed linear subspace. A Banach space is said to be hereditarily indecomposable (H.I.) if for every pair $Y, Z$ of subspaces of $X$ with $Y \cap Z=\{\mathbf{0}\}$, the subspace $Y+Z$ is not closed. The famous example of Gowers and Maurey [15] of a Banach space without unconditional basic sequence was observed by W. Johnson to be H.I. Since the appearance of the Gowers-Maurey space the study of H.I. spaces has been one of the most important research topics in modern Banach space theory. We refer to [23] and $[6]$ for a detailed survey of results.

Gower's remarkable dichotomy [14] states that every Banach space either contains an infinite unconditional sequence, or a subspace which is H.I. It is proved in [6] that every Banach space not containing an isomorph of $\ell_{1}$ has a subspace which is a quotient of an H.I. space. A recent result of S. Argyros [3] states that a separable Banach space universal for the class of reflexive H.I. spaces, is also universal for the class of separable Banach spaces. These results indicate the large variety of H.I. spaces.

The construction of H.I. spaces is not an easy task. The crucial step was Schlumprecht's construction of an arbitrarily distortable Banach space [27]. Recall that the Banach space $(X,\|\cdot\|)$ is arbitrarily distortable if for every $\lambda>1$, there exists an equivalent norm $|\cdot|$ on $X$ so that for every subspace $Y$ of $X$ there exist non-zero vectors $x, y$ in $Y$ such that $\|x\|=$ $\|y\|$, yet $|x| /|y|>\lambda$. Schlumprecht's space had an immense impact on the

2000 Mathematics Subject Classification: Primary 46B03; Secondary 06A07, 03E02.

Key words and phrases: distortion, hereditarily indecomposable space, Tsirelson's space, Schreier sets. 
development of the theory because of its connection to the Gowers-Maurey construction, as well as to the solution of the distortion problem for $\ell_{p}$, $1<p<\infty$ [24]. It is proven in [29] that every H.I. space is arbitrarily distortable.

The first example of an arbitrarily distortable, asymptotic $\ell_{1}$ space was given in [4]. We recall that a Banach space with a normalized basis $\left(e_{n}\right)$ is asymptotic $\ell_{1}[19]$ if there exists a constant $C>0$ such that for every $k \in \mathbb{N}$ there exists $N \in \mathbb{N}$ such that every sequence $\left(x_{i}\right)_{i=1}^{k}$ of successive normalized blocks of $\left(e_{n}\right)$ is $C$-equivalent to the canonical basis of $\ell_{1}^{k}$. It was shown in [4] that there exist infinite subsets $M=\left(m_{i}\right), N=\left(n_{i}\right)$ of $\mathbb{N}$ so that the mixed Tsirelson space $T\left(1 / m_{i}, S_{n_{i}}\right)_{i=1}^{\infty}$ is arbitrarily distortable. In the same paper this example was conditionalized to yield an asymptotic $\ell_{1}$ H.I. space.

The main goal of this paper is to find a more conceptual approach to a certain class of asymptotic $\ell_{1}$ H.I. spaces. More precisely we show the following

THEOREM 1.1. There exists a family of cardinality equal to the continuum whose members are totally incomparable, asymptotic $\ell_{1}$, reflexive H.I. spaces.

Recall that the Banach spaces $X$ and $Y$ are totally incomparable if no subspace of $X$ is isomorphic to a subspace of $Y$. The proof of Theorem 1.1 is based on ideas from [4]. However, our argument is considerably simpler.

We now describe how this paper is organized. In Section 3 we introduce, for a given scalar $d>1$, infinite subsets $N$ and $P$ of $\mathbb{N}$ and a null scalar sequence $\mathbf{a}$, the $(d, N, P, \mathbf{a})$ distortion property (Definition 3.1) for a certain class of asymptotic $\ell_{1}$ Banach spaces. This property, which roughly speaking is related to the optimality of the constants of higher order $\ell_{1}$-spreading models of the space, will enable us to give a criterion (Theorem 3.2) for certain asymptotic $\ell_{1}$ Banach spaces to be arbitrarily distortable. We also show how to obtain totally incomparable arbitrarily distortable spaces.

We apply Theorem 3.2 in Section 4 in order to give an alternative proof of the fact that certain mixed Tsirelson spaces are arbitrarily distortable [4], [2], [5]. These spaces can be described as the completion of $c_{00}$, the space of all ultimately vanishing real sequences, under the norm given by $\|x\|=\sup \left\{\sum_{i=1}^{\infty} \mu(\{i\}) x(i): \mu \in \mathcal{M}\right\}$, where $\mathcal{M}$ is a suitable symmetric subset of the finitely supported signed measures on $\mathbb{N}$ containing the point mass measures and closed under interval restrictions. The main difficulty in the study of mixed Tsirelson spaces is that the norming set $\mathcal{M}$ is defined by means of an inductive procedure. We are able to bypass this difficulty by describing $\mathcal{M}$ analytically and proving a decomposition result for its members (Lemma 4.6), which greatly simplifies the argument for the distortion of $T\left(1 / m_{i}, S_{n_{i}}\right)_{i=1}^{\infty}$. 
In Section 5, we choose a subset $\mathcal{N}$ of $\mathcal{M}$ which is maximal with respect to a Maurey-Rosenthal type of condition [20] and show in Theorem 3.5 that the completion of $c_{00}$ under the norm induced by $\mathcal{N}$ is an H.I. space with the $(d, N, P, \mathbf{a})$ distortion property. Various choices of $\mathcal{N}$ give rise to totally incomparable H.I. spaces.

In order to prove that a space $X$ is H.I., we employ Theorem 3.6 which loosely speaking asserts that if for every $\varepsilon>0$ there exist integers $k<n$ such that every block subspace $Y$ of $X$ contains a sufficiently large (in the Schreier sense) block basis $z_{1}<\ldots<z_{p}$ with the property that $\left\|\sum_{i=1}^{p} a_{i} z_{i}\right\| \geq$ $\varepsilon\left\|\sum_{i=1}^{p} a_{i} e_{i}\right\|_{n}$ whenever $\left(a_{i}\right)_{i=1}^{p} \subset \mathbb{R}^{+}$, while $\left\|\sum_{i=1}^{p} a_{i} z_{i}\right\| \leq\left\|\sum_{i=1}^{p} a_{i} e_{i}\right\|_{C k}$ for every sequence $\left(a_{i}\right)_{i=1}^{p}$ in $\mathbb{R}$, then $X$ contains no infinite unconditional sequence. Above, $\left(e_{i}\right)$ is the natural unit vector basis of $c_{00}$ and $\|\cdot\|_{n},\|\cdot\|_{C k}$ denote the $n$th Schreier and $k$ th conditional Schreier norms respectively.

The precise statements for the results mentioned above are given in Section 3. The proof of Theorem 1.1, presented in Section 3, follows from Theorem 3.5 and Proposition 3.3 combined with two fundamental results of descriptive set theory, the infinite Ramsey theorem [10], [22] and a theorem of Kuratowski [17].

Acknowledgments. I wish to thank the referee for correcting an error in an earlier version of this paper, and for useful comments and suggestions regarding the material discussed herein.

2. Preliminaries. We shall make use of standard Banach space facts and terminology as may be found in [18]. If $D$ is any set, we let $[D]$ (resp. $[D]^{<\infty}$ ) denote the set of its infinite (resp. finite) subsets. Given $M \in[\mathbb{N}]$, the notation $M=\left(m_{i}\right)$ indicates that $M=\left\{m_{1}<m_{2}<\ldots\right\}$. Let $E$ and $F$ be finite subsets of $\mathbb{N}$. We write $E<F$ if $\max E<\min F$.

Suppose now that $X$ is a Banach space with a Schauder basis $\left(e_{n}\right)$. A sequence $\left(u_{n}\right)$ of non-zero vectors in $X$ is a block basis of $\left(e_{n}\right)$ if there exist successive subsets $F_{1}<F_{2}<\ldots$ of $\mathbb{N}$ and a scalar sequence $\left(a_{n}\right)$ so that $u_{n}=\sum_{i \in F_{n}} a_{i} e_{i}$ for every $n \in \mathbb{N}$. We adopt the notation $u_{1}<u_{2}<\ldots$ to indicate that $\left(u_{n}\right)$ is a block basis of $\left(e_{n}\right)$. We let $\operatorname{supp} u_{n}$ denote the set $\left\{i \in F_{n}: a_{i} \neq 0\right\}$. The range $r\left(u_{n}\right)$ of $u_{n}$ is the smallest integer interval containing supp $u_{n}$. The subspace of $X$ generated by a block basis of $\left(e_{n}\right)$ is called a block subspace.

We next review two important hierarchies: the Schreier hierarchy $\left\{S_{\xi}\right\}_{\xi<\omega_{1}}$ (see [1]) and the repeated averages hierarchy, $\left(\xi_{n}^{M}\right)_{n=1}^{\infty}, \xi<\omega_{1}$, $M \in\left[\mathbb{N}\right.$ ] (see [8]). Since we shall only be using the families $\left\{S_{\xi}\right\}_{\xi<\omega}$ and $\left(\xi_{n}^{M}\right)_{n=1}^{\infty}, \xi<\omega, M \in[\mathbb{N}]$, we confine the definitions to the finite ordinal case. 
The Schreier families. We let $S_{0}=\{\{n\}: n \in \mathbb{N}\} \cup\{\emptyset\}$. Suppose $S_{\xi}$ has been defined, $\xi<\omega$. We set

$$
\begin{aligned}
S_{\xi+1}=\left\{\bigcup_{i=1}^{n} F_{i}: n \in \mathbb{N},\right. & n \leq \\
& \min F_{1}, \\
& \left.F_{1}<\ldots<F_{n}, F_{i} \in S_{\xi}(i \leq n)\right\} \cup\{\emptyset\} .
\end{aligned}
$$

An important property shared by the Schreier families is that they are hereditary: If $F \in S_{\xi}$ and $G \subset F$, then $G \in S_{\xi}$. Another important property is that they are spreading: If $\left\{p_{1}, \ldots, p_{k}\right\} \in S_{\xi}, p_{1}<\ldots<p_{k}$, and $q_{1}<\ldots<q_{k}$ are so that $p_{i} \leq q_{i}$ for all $i \leq k$, then $\left\{q_{1}, \ldots, q_{k}\right\} \in S_{\xi}$. It is not hard to verify the following convolution property of Schreier families: if $F_{1}<\ldots<F_{n}$ are members of $S_{\alpha}$ such that $\left\{\min F_{i}: i \leq n\right\}$ belongs to $S_{\beta}$, then $\bigcup_{i=1}^{n} F_{i}$ belongs to $S_{\alpha+\beta}$.

The repeated averages hierarchy. We first let $\left(e_{n}\right)$ denote the unit vector basis of $c_{00}$. Given $\xi<\omega$ and $M \in[\mathbb{N}]$, we define by induction a sequence $\left(\xi_{n}^{M}\right)_{n=1}^{\infty}$ of finitely supported probability measures on $\mathbb{N}$ whose supports are successive subsets of $M$.

If $\xi=0$, then $\xi_{n}^{M}=e_{m_{n}}$ for all $n \in \mathbb{N}$, where $M=\left(m_{n}\right)$.

Assume that $\left(\xi_{n}^{M}\right)_{n=1}^{\infty}$ has been defined for all $M \in[\mathbb{N}]$. Set

$$
[\xi+1]_{1}^{M}=\frac{1}{m_{1}} \sum_{i=1}^{m_{1}} \xi_{i}^{M}
$$

where $m_{1}=\min M$. Suppose that $[\xi+1]_{1}^{M}<\ldots<[\xi+1]_{n}^{M}$ have been defined. Let

$$
M_{n}=\left\{m \in M: m>\max \operatorname{supp}[\xi+1]_{n}^{M}\right\} \quad \text { and } \quad k_{n}=\min M_{n} .
$$

Set

$$
[\xi+1]_{n+1}^{M}=\frac{1}{k_{n}} \sum_{i=1}^{k_{n}} \xi_{i}^{M_{n}} .
$$

It follows that $\operatorname{supp} \xi_{n}^{M}$ belongs to $S_{\xi}$, and moreover it is a maximal (under inclusion) member of $S_{\xi}$. It can be easily shown, by induction, that if $i$ and $j$ belong to $\operatorname{supp} \xi_{n}^{M}$ and $i<j$, then $\xi_{n}^{M}(\{i\}) \geq \xi_{n}^{M}(\{j\})$.

For a probability measure $\mu$ in $\mathbb{N}$ and $\xi<\omega$, define $\|\mu\|_{\xi}=\sup \{\mu(F)$ : $\left.F \in S_{\xi}\right\}$. It is proven in [13], [7] that $\left\|\xi_{1}^{M}\right\|_{\xi-1} \leq \xi / \min M$ for every $\xi \geq 1$ and $M \in[\mathbb{N}]$. It follows that for every $P \in[\mathbb{N}]$, every $\xi \geq 1$ and every $\varepsilon>0$, there exists $M \in[P]$ such that $\left\|\xi_{1}^{M}\right\|_{\xi-1}<\varepsilon$. This property of the repeated averages will be very useful in what follows. For a detailed study of these hierarchies we refer to [1], [8], [26], [12], [7] and [13].

We continue by introducing some more terminology. A finite collection $\mathcal{F}$ of finite subsets of $\mathbb{N}$ is said to be $r S_{\xi^{-}}$admissible, $\xi<\omega, r \in \mathbb{N}$, if there 
exists an enumeration $\left\{I_{k}: k \leq n\right\}$ of $\mathcal{F}$ such that $I_{1}<\ldots<I_{n}$ and the set $\left\{\min I_{k}: k \leq n\right\}$ is the union of $r$ members of $S_{\xi}$. In case $\left\{\min I_{k}: k \leq n\right\}$ is a maximal (under inclusion) member of $S_{\xi}, \mathcal{F}$ is called maximally $S_{\xi^{-}}$ admissible. A finite block basis $u_{1}<\ldots<u_{n}$ in a Banach space with a basis is $r S_{\xi}$ (resp. maximally $S_{\xi}$ )-admissible if $\left\{\operatorname{supp} u_{i}: i \leq n\right\}$ is.

In what follows, $X$ is a Banach space with a basis $\left(e_{n}\right)$. The support of every block basis of $\left(e_{n}\right)$ will always be taken with respect to $\left(e_{n}\right)$.

DeFinition 2.1. Let $\left(u_{n}\right)$ be a normalized block basis of $\left(e_{n}\right), \varepsilon>0$ and $1 \leq \xi<\omega$. Set $p_{n}=\min \operatorname{supp} u_{n}, n \in \mathbb{N}$, and $P=\left(p_{n}\right)$.

(1) A generic $(\varepsilon, \xi)$ average of $\left(u_{n}\right)$ is any vector that can be written in the form $\sum_{n=1}^{\infty} \xi_{1}^{R}\left(p_{n}\right) u_{n}$, where $R \in[P]$ and $\left\|\xi_{1}^{R}\right\|_{\xi-1}<\varepsilon$.

(2) An $(\varepsilon, \xi)$ average of $\left(u_{n}\right)$ is any generic $(\varepsilon, \xi)$ average of a normalized block basis of $\left(u_{n}\right)$.

(3) A normalized $(\varepsilon, \xi)$ average of $\left(u_{n}\right)$ is any vector $u$ of the form $u=$ $v /\|v\|$, where $v$ is an $(\varepsilon, \xi)$ average of $\left(u_{n}\right)$. In case $\|v\| \geq 1 / 2, u$ is a smoothly normalized $(\varepsilon, \xi)$ average of $\left(u_{n}\right)$.

Notation. Let $E^{*}$ be a finite collection of successive intervals of $\mathbb{N}$ and let $u$ be a finite linear combination of $\left(e_{n}\right)$.

(1) We let $I\left(u, E^{*}\right)$ denote the number of elements of $E^{*}$ which intersect $\operatorname{supp} u$.

(2) Let $D$ be a finite block basis of $\left(e_{n}\right)$ such that the support of every member of $D$ intersects at least one member of $E^{*}$. We define $D\left(E^{*}, 1\right)=$ $\left\{u \in D: I\left(u, E^{*}\right)=1\right\}$ and $D\left(E^{*}, 2\right)=\left\{u \in D: I\left(u, E^{*}\right) \geq 2\right\}$.

REMARK 2.2. Let $E^{*}$ be an $S_{p}$-admissible collection of intervals of $\mathbb{N}$. Let $D$ be a finite block basis of $\left(e_{n}\right)$ such that the support of every member of $D$ intersects at least one member of $E^{*}$. Given $J \in E^{*}$ denote by $D(J)$ the collection of those $u \in D$ for which $J$ is the only member of $E^{*}$ intersecting $\operatorname{supp} u$. Assume that for every $J \in E^{*}, D(J)$ is $r S_{q}$-admissible. The spreading property of the Schreier families implies that $D\left(E^{*}, 2\right)$ is $2 S_{p}$-admissible. On the other hand, the convolution and spreading properties of Schreier families imply that $D\left(E^{*}, 1\right)$ is $(r+1) S_{p+q}$-admissible and thus $D$ is $(r+3) S_{p+q}$-admissible.

Before closing this section, we recall the definitions of the Schreier space, $X^{\xi}$, and conditional Schreier space, $\mathrm{C} X^{\xi}, \xi<\omega . X^{\xi}$ is the completion of $c_{00}$ under the norm $\|x\|_{\xi}=\sup \left\{\sum_{i \in F}|x(i)|: F \in S_{\xi}\right\} . X^{0}$ is isometric to $c_{0} . X^{1}$ was introduced by Schreier [28] in order to provide an example of a weakly null sequence without Cesàro summable subsequence. The generalized family $\left\{X^{\xi}\right\}_{\xi<\omega_{1}}$ of Schreier spaces was studied in [1], where it is shown that the natural Schauder basis $\left(e_{n}\right)$ of $X^{\xi}$ is 1-unconditional and shrinking. For a detailed study of the spaces $\left\{X^{\xi}\right\}_{\xi<\omega}$ we refer to [13]. 
The conditional Schreier spaces $\left\{\mathrm{C} X^{\xi}\right\}_{\xi<\omega}$ were constructed by H. Rosenthal (unpublished). $\mathrm{C} X^{\xi}$ is the completion of $c_{00}$ under the norm

$$
\|x\|_{\mathrm{C} \xi}=\sup \left\{\sum_{k=1}^{n}\left|\sum_{i \in J_{k}} x(i)\right|: n \in \mathbb{N},\right.
$$

$$
\left.\left(J_{k}\right)_{k=1}^{n} \text { are } S_{\xi^{-}} \text {admissible intervals }\right\} \text {. }
$$

The natural basis $\left(e_{n}\right)$ of $\mathrm{C} X^{\xi}$ is of course a conditional basis. When $\xi=0$, $\left(e_{n}\right)$ is equivalent to the summing basis of $c_{0}$. We also mention the following useful fact: Suppose $\left(a_{i}\right)_{i=1}^{n}$ is a non-increasing finite sequence of non-negative scalars. Then $\left\|\sum_{i=1}^{n}(-1)^{i} a_{i} e_{t_{i}}\right\|_{\mathrm{C} \xi} \leq\left\|\sum_{i=1}^{n} a_{i} e_{t_{i}}\right\|_{\xi}$ for every increasing sequence $\left(t_{i}\right)_{i=1}^{n}$ of integers.

3. Main results. We start this section by recalling that a normalized sequence $\left(x_{n}\right)$ in a Banach space is an $\varepsilon-\ell_{1}^{\xi}$ spreading model, $\varepsilon>0$, if $\left\|\sum_{i \in F} a_{i} x_{i}\right\| \geq \varepsilon \sum_{i \in F}\left|a_{i}\right|$ for every $F \in S_{\xi}$ and all choices of scalars $\left(a_{i}\right)_{i \in F}$.

A basis $\left(e_{n}\right)$ for a Banach space $X$ is said to have property $\varepsilon-\ell_{1}^{\xi}, 1 \leq \xi$

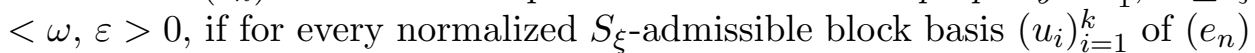
we have $\left\|\sum_{i=1}^{k} a_{i} u_{i}\right\| \geq \varepsilon \sum_{i=1}^{k}\left|a_{i}\right|$ for all choices of scalars $\left(a_{i}\right)_{i=1}^{k}$. Clearly, $X$ is then asymptotic $\ell_{1}$.

The spreading property of $S_{\xi}$ implies that if $\left(e_{n}\right)$ has property $\varepsilon-\ell_{1}^{\xi}$, then so do all of its block bases. The spreading property of $S_{\xi}$ also implies that every normalized block basis of $\left(e_{n}\right)$ is an $\varepsilon-\ell_{1}^{\xi}$ spreading model.

For a Banach space $X$ with a basis $\left(e_{n}\right)$ having property $\varepsilon-\ell_{1}^{\xi}$ and $\delta>0$, we define

$$
\begin{array}{r}
\tau\left(\left(e_{n}\right), \delta\right)=\sup \left\{\zeta<\omega \text { : every normalized block basis of }\left(e_{n}\right)\right. \\
\text { has a subsequence which is a } \left.\delta-\ell_{1}^{\zeta} \text { spreading model }\right\} .
\end{array}
$$

Evidently, $\tau\left(\left(e_{n}\right), \varepsilon\right) \geq \xi$. The modulus $\tau\left(\left(e_{n}\right), \delta\right)$ is implicitly defined in [26] and $[2]$.

Definition 3.1. Let $X$ be a Banach space with a basis $\left(e_{i}\right)$. Let $N=$ $\left(n_{i}\right)$ and $P=\left(p_{i}\right)$ be infinite subsets of $\mathbb{N}$ such that $n_{i-1} \leq p_{i}<n_{i} / 2$ for every $i \in \mathbb{N}$. Let $\mathbf{a}=\left(\delta_{i}\right)$ be a decreasing null sequence of scalars, and let $d>1$. Then $X$ is said to have the $(d, N, P, \mathbf{a})$ distortion property if for every $j \in \mathbb{N},\left(e_{i}\right)$ has property $\delta_{j}-\ell_{1}^{n_{j}}$, while $\tau\left(\left(u_{i}\right), d \delta_{j}\right)<p_{j}$ for every normalized block basis $\left(u_{i}\right)$ of $\left(e_{i}\right)$.

THEOREM 3.2. Let $(X,\|\cdot\|)$ be a Banach space with a normalized, shrinking, bimonotone basis $\left(e_{i}\right)$. Suppose that there exist $N, P$ in $[\mathbb{N}]$, a scalar sequence $\mathbf{a}=\left(\delta_{i}\right)$ and $d>1$ so that $X$ has the $(d, N, P, \mathbf{a})$ distortion property. Then $X$ is arbitrarily distortable. 
Proof. In what follows, the admissibility of every block basis of $\left(e_{i}\right)$ will always be considered with respect to $\left(e_{i}\right)$. Given $j \in \mathbb{N}$, we set

$$
\mathcal{A}_{j}=\left\{\delta_{j} \sum_{i=1}^{k} x_{i}^{*}:\left(x_{i}^{*}\right)_{i=1}^{k} \subset B_{X^{*}} \text { is } S_{n_{j}} \text {-admissible }\right\} .
$$

Above, the admissibility of $\left(x_{i}^{*}\right)_{i=1}^{k}$ is measured with respect to $\left(e_{i}^{*}\right)$, the sequence of functionals biorthogonal to $\left(e_{i}\right)$. Because $\left(e_{i}\right)$ has property $\delta_{j^{-}}$ $\ell_{1}^{n_{j}}$, we deduce that $\mathcal{A}_{j} \subset B_{X^{*}}$. Indeed, suppose that $\delta_{j} \sum_{i=1}^{k} x_{i}^{*} \in \mathcal{A}_{j}$ and let $x \in X$ with $\|x\| \leq 1$.

Set $J_{i}=\left[\min \operatorname{supp} x_{i}^{*}, \min \operatorname{supp} x_{i+1}^{*}\right)$ for $i \leq k-1$, and $J_{k}=r\left(x_{k}^{*}\right)$. Define $x_{i}=x \mid J_{i}, i \leq k$. Since $\left(e_{i}\right)$ is bimonotone, $\left\|\sum_{i=1}^{k} x_{i}\right\| \leq 1$. Furthermore, $\left(x_{i}\right)_{i=1}^{k}$ is $S_{n_{j}}$-admissible. Hence, $\delta_{j} \sum_{i=1}^{k}\left\|x_{i}\right\| \leq 1$ and the assertion follows.

We define an equivalent norm $\|\cdot\|_{j}$ on $X$ in the following manner:

$$
\|x\|_{j}=\sup \left\{x^{*}(x): x^{*} \in \mathcal{A}_{j}\right\} .
$$

We are going to show that for every normalized block basis $\left(u_{i}\right)$ of $\left(e_{i}\right)$ and all $j \in \mathbb{N}$ there exists a finite linear combination $w_{0}$ of $\left(u_{i}\right)$ such that $\left\|w_{0}\right\|=1,\left\|w_{0}\right\|_{j} \geq 1 /(8 d+1)$ and $\left\|w_{0}\right\|_{j_{0}} \leq(8 d+4) \delta_{j_{0}}$ for all $j_{0}<j$.

Once this is accomplished, given a normalized block basis $\left(u_{i}\right)$ of $\left(e_{i}\right)$ and $j_{0}<j$, choose finite linear combinations $v_{0}$ and $w_{0}$ of $\left(u_{i}\right)$ such that $\left\|v_{0}\right\|=1,\left\|v_{0}\right\|_{j_{0}} \geq 1 /(8 d+1)$, while $\left\|w_{0}\right\|=1$ and $\left\|w_{0}\right\|_{j_{0}} \leq(8 d+4) \delta_{j_{0}}$. It follows that

$$
\frac{\left\|v_{0}\right\|_{j_{0}}}{\left\|w_{0}\right\|_{j_{0}}} \geq \frac{1}{(8 d+1)(8 d+4) \delta_{j_{0}}}
$$

Since $j_{0}$ is arbitrary we conclude that $X$ is arbitrarily distortable.

We first show that for every normalized block basis $\left(u_{i}\right)$ of $\left(e_{i}\right)$ and $j \in \mathbb{N}$, there exists a normalized block basis $\left(w_{i}\right)$ of $\left(u_{i}\right)$ such that for every $x^{*} \in B_{X^{*}}$, the block basis $V_{x^{*}}=\left\{w_{i}: i \in \mathbb{N},\left|x^{*}\left(w_{i}\right)\right| \geq 8 d \delta_{j}\right\}$ is $S_{p_{j}}$-admissible.

Indeed, since $\tau\left(\left(u_{i}\right), d \delta_{j}\right)<p_{j}$, there exists a normalized block basis $\left(v_{i}\right)$ of $\left(u_{i}\right)$ having no subsequence which is a $d \delta_{j}-\ell_{1}^{p_{j}}$ spreading model. Set $Q=\left(q_{i}\right)$ where $q_{i}=\min \operatorname{supp} v_{i}$ for all $i \in \mathbb{N}$. Define

$$
\mathcal{F}_{j}=\left\{G \in[Q]^{<\infty}: \exists x^{*} \in B_{X^{*}},\left|x^{*}\left(v_{i}\right)\right| \geq 8 d \delta_{j}, \forall i \in \mathbb{N}\left(q_{i} \in G\right)\right\} .
$$

Of course, $\mathcal{F}_{j}$ is hereditary. We claim that there exists $R \in[Q]$ such that $\mathcal{F}_{j} \cap[R]^{<\infty} \subset S_{p_{j}}$. If that were not the case, then by the result of [12] there exists $R \in[Q]$ such that $S_{p_{j}} \cap[R]^{<\infty} \subset \mathcal{F}_{j}$. Suppose $R=\left(q_{k_{i}}\right)$. Then given $F \in S_{p_{j}}$ there exists $x^{*} \in B_{X^{*}}$ so that $\left|x^{*}\left(v_{k_{i}}\right)\right| \geq 8 d \delta_{j}$ for all $i \in F$. Corollary 3.6 of [7] now yields a subsequence of $\left(v_{k_{i}}\right)$ which is a $d \delta_{j}-\ell_{1}^{p_{j}}$ spreading model, contradicting our assumption on $\left(v_{i}\right)$. Therefore, our claim holds and if we set $w_{i}=v_{k_{i}}\left(R=\left(q_{k_{i}}\right)\right)$, then $\left(w_{i}\right)$ is a normalized block 
basis of $\left(u_{i}\right)$ such that $V_{x^{*}}=\left\{w_{i}: i \in \mathbb{N},\left|x^{*}\left(w_{i}\right)\right| \geq 8 d \delta_{j}\right\}$ is $S_{p_{j}}$-admissible for all $x^{*} \in B_{X^{*}}$.

We next choose a generic $\left(\delta_{j}^{2}, n_{j}\right)$ average $w$ of $\left(w_{i}\right)$. It is clear that for some $y_{j}^{*} \in \mathcal{A}_{j}$ we have $y_{j}^{*}(w) \geq \delta_{j}$, and thus $\|w\|_{j} \geq \delta_{j}$. Since $V_{x^{*}}$ is $S_{p_{j}}$ admissible for every $x^{*} \in B_{X^{*}}$ and $p_{j}<n_{j}$, we see that $\left|x^{*}(w)\right| \leq 8 d \delta_{j}+\delta_{j}^{2}$ for all $x^{*} \in B_{X^{*}}$ and therefore $\|w\| \leq(8 d+1) \delta_{j}$. We have thus shown that $\delta_{j} \leq\|w\| \leq(8 d+1) \delta_{j}$ and $\|w\|_{j} \geq \delta_{j}$.

Suppose now that $j_{0}<j$ and let $\delta_{j_{0}} \sum_{r=1}^{k} x_{r}^{*} \in \mathcal{A}_{j_{0}}$. Set $D=\left\{w_{i}\right.$ : $\left.\left|\sum_{r=1}^{k} x_{r}^{*}\left(w_{i}\right)\right| \geq 8 d \delta_{j}\right\}$ and let $E^{*}$ denote the collection of the ranges of the $x_{r}^{*}$ 's. Since $V_{x^{*}}$ is $S_{p_{j}}$-admissible for every $x^{*} \in B_{X^{*}}$, Remark 2.2 implies that $D\left(E^{*}, 1\right)$ is $2 S_{n_{j_{0}}+p_{j}}$-admissible. On the other hand $D\left(E^{*}, 2\right)$ is $2 S_{n_{j_{0}}}$ admissible and thus $D$ is $4 S_{2 p_{j}}$-admissible as $n_{j_{0}} \leq p_{j}$. Because $2 p_{j}<n_{j}$, we obtain the estimate

$$
\left|\delta_{j_{0}} \sum_{r=1}^{k} x_{r}^{*}(w)\right| \leq 8 d \delta_{j} \delta_{j_{0}}+4 \delta_{j}^{2} .
$$

Hence, $\|w\|_{j_{0}} \leq 8 d \delta_{j} \delta_{j_{0}}+4 \delta_{j}^{2}$. If we set $w_{0}=w /\|w\|$, then $\left\|w_{0}\right\|_{j} \geq 1 /(8 d+1)$ since $\|w\|_{j} \geq \delta_{j}$ and $\|w\| \leq(8 d+1) \delta_{j}$, while $\left\|w_{0}\right\|_{j_{0}} \leq(8 d+4) \delta_{j_{0}}$ since $\|w\|_{j_{0}} \leq 8 d \delta_{j} \delta_{j_{0}}+4 \delta_{j}^{2},\|w\| \geq \delta_{j}$ and $j_{0}<j$. This completes the entire proof.

Proposition 3.3. Let $X_{r}$ have a shrinking basis $\left(e_{k}^{r}\right)_{k=1}^{\infty}, r=1,2$. Assume that $X_{r}$ has the $\left(d_{r}, N_{r}, P_{r}, \mathbf{a}\right)$ distortion property, $r=1,2$, and that $\mathbf{a}=\left(\delta_{i}\right)$ satisfies $\lim _{i} \delta_{i+1} / \delta_{i}=0$. Suppose that for every $i_{0} \in \mathbb{N}$ there exist $i>j>i_{0}$ such that $n_{i}^{1}=n_{j}^{2}$, where $N_{r}=\left(n_{k}^{r}\right)_{k=1}^{\infty}, r=1,2$. Then $X_{1}$ and $\mathrm{X}_{2}$ are totally incomparable.

Proof. Suppose the assertion is false. A standard perturbation argument yields a normalized block basis $\left(u_{k}\right)$ of $\left(e_{k}^{1}\right)$ equivalent to a block basis $\left(w_{k}\right)$ of $\left(e_{k}^{2}\right)$. Let $T$ be an isomorphism from $\left[\left(u_{k}\right)\right]$ onto $\left[\left(w_{k}\right)\right]$ such that $T\left(u_{k}\right)=w_{k}$, for all $k \in \mathbb{N}$. We can choose $i_{0} \in \mathbb{N}$ such that

$$
\frac{\delta_{i+1}}{\delta_{i}}<\frac{1}{d_{1}\|T\| \cdot\left\|T^{-1}\right\|} \quad \text { for every } i \geq i_{0} .
$$

Our assumptions allow us to choose $i>j>i_{0}$ such that $n_{i}^{1}=n_{j}^{2}$. Let $\left(v_{k}\right)$ be a normalized block basis of $\left(u_{k}\right)$ having no subsequence which is a $d_{1} \delta_{i}-\ell_{1}^{n_{i}^{1}}$ spreading model. But since $\left(T\left(v_{k}\right)\right)$ is a block basis of $\left(w_{k}\right)$, it follows that for every $F \in S_{n_{j}^{2}}$ and all choices of scalars $\left(a_{k}\right)_{k \in F}$,

$$
\left\|\sum_{k \in F} a_{k} T\left(v_{k}\right)\right\| \geq \frac{\delta_{j}}{\left\|T^{-1}\right\|} \sum_{k \in F}\left|a_{k}\right| .
$$


Hence,

$$
\left\|\sum_{k \in F} a_{k} v_{k}\right\| \geq \frac{\delta_{j}}{\|T\| \cdot\left\|T^{-1}\right\|} \sum_{k \in F}\left|a_{k}\right|
$$

for every $F \in S_{n_{j}^{2}}$ and all choices of scalars $\left(a_{k}\right)_{k \in F}$. However, $\delta_{i} / \delta_{j} \leq$ $\delta_{j+1} / \delta_{j}$, and therefore $\delta_{j} /\left(\|T\| \cdot\left\|T^{-1}\right\|\right)>d_{1} \delta_{i}$. Thus, $\left(v_{k}\right)$ is a $d_{1} \delta_{i}-\ell_{1}^{n_{i}^{1}}$ spreading model contrary to our assumptions.

Definition 3.4. Let $M=\left(m_{i}\right) \in[\mathbb{N}]$ be such that $m_{1}>7$ and $m_{i}^{2}<$ $m_{i+1}$ for all $i \in \mathbb{N}$. Choose $L=\left(l_{i}\right) \in[\mathbb{N}]$ such that $l_{1}>4$ and $2^{l_{i}}>m_{i}$ for all $i \in \mathbb{N}$. The infinite subset $N=\left(n_{i}\right)$ of $\mathbb{N}$ is said to be $M$-good if $l_{j}\left(f_{j}^{N}+1\right)<n_{j}$ for all $j \in \mathbb{N}$, where $\left(f_{j}^{N}\right)$ is the sequence given by $f_{1}^{N}=1$ while for $j \geq 2$,

$$
f_{j}^{N}=\max \left\{\sum_{i<j} \varrho_{i} n_{i}: \varrho_{i} \in \mathbb{N} \cup\{0\}(i<j), \prod_{i<j} m_{i}^{\varrho_{i}}<m_{j}^{3}\right\} .
$$

Note that $f_{j}^{N}$ is well defined because $m_{1}>1$. It is easy to see that for every $P \in[\mathbb{N}]$ there exists $N \in[P]$ which is $M$-good. The main result of Section 5 is the following

Theorem 3.5. Suppose $N=\left(n_{i}\right)$ is $M$-good. Set $N^{(2)}=\left(n_{2 i}\right), F^{(2)}=$ $\left(f_{2 i}^{N}+2\right)$ and $\mathbf{a}=\left(1 / m_{2 i}\right)$. Then there exists a reflexive H.I. space $X(N)$ with the $\left(6, N^{(2)}, F^{(2)}, \mathbf{a}\right)$ distortion property.

The proof is given in Section 5. We now pass to the

Proof of Theorem 1.1. We first choose $N_{0} \in[\mathbb{N}]$ such that every $N \in\left[N_{0}\right]$ is $M$-good. To see that such an $N_{0}$ exists, set

$$
\mathcal{D}=\{N \in[\mathbb{N}]: N \text { is } M \text {-good }\} .
$$

We can easily verify that $\mathcal{D}$ is closed in the topology of pointwise convergence in $[\mathbb{N}]$, and therefore it is a Ramsey set. Because $\mathcal{D} \cap[R] \neq \emptyset$ for every $R \in[\mathbb{N}]$, the infinite Ramsey theorem yields $N_{0} \in[\mathbb{N}]$ such that $\left[N_{0}\right] \subset \mathcal{D}$, as claimed.

It is a well known fact that $\left[N_{0}\right]$ endowed with the topology of pointwise convergence is a perfect Polish space. We let $\left[N_{0}\right]^{2}=\left[N_{0}\right] \times\left[N_{0}\right]$ and set $G=\left\{(N, R) \in\left[N_{0}\right]^{2}: N=\left(n_{i}\right), R=\left(r_{i}\right), \forall i_{0} \in \mathbb{N}, \exists i>j>i_{0}: n_{2 i}=r_{2 j}\right\}$. A straightforward application of the Baire category theorem shows that $G$ is a dense $G_{\delta}$ subset of $\left[N_{0}\right] \times\left[N_{0}\right]$. We recall here a special case of a result of Kuratowski [17] and Mycielski [21] (cf. [16], p. 129, Theorem 19.1, or Proposition 3.6 of [13]) which asserts that for a perfect Polish space $K$ and a dense $G_{\delta}$ subset $G$ of $K \times K$, there exists a subset $C$ of $K$ homeomorphic to the Cantor set and such that $C \times C \backslash \Delta \subset G$ ( $\Delta$ stands for the diagonal of $K \times K)$. 
It follows from this result that there exists $C \subset\left[N_{0}\right]$ homeomorphic to the Cantor set such that $\left(N_{1}, N_{2}\right) \in G$ whenever $N_{1}, N_{2}$ are distinct elements of $C$.

We can now apply Theorem 3.5 to obtain a family $\{X(N): N \in C\}$ of reflexive H.I. spaces such that for every $N \in C, X(N)$ has the $\left(6, N^{(2)}, F^{(2)}, \mathbf{a}\right)$ distortion property, where $N^{(2)}, F^{(2)}$ and a are as in the statement of Theorem 3.5. Since $\left(N_{1}, N_{2}\right) \in G$ whenever $N_{1}$ and $N_{2}$ are distinct elements of $C$, Proposition 3.3 implies that $X\left(N_{1}\right)$ and $X\left(N_{2}\right)$ are totally incomparable. The proof of the theorem is now complete.

To construct H.I. spaces we shall make use of the following

TheOrem 3.6. Let $X$ be a Banach space with a basis $\left(x_{i}\right)$. Let $\left(n_{j}\right),\left(k_{j}\right)$ be increasing sequences of positive integers such that $k_{j}<n_{j}$ for all $j \in \mathbb{N}$, and let $\left(\delta_{j}\right)$ be a null sequence of positive scalars. Assume that there exist absolute positive constants $c_{1}, c_{2}, c_{3}$ such that for every block subspace $Y$ of $X$ and every $j \in \mathbb{N}$ there exists a block basis $z_{1}<\ldots<z_{p}$ of $\left(x_{i}\right)$ in $Y$ such that letting $t_{i}=\min \operatorname{supp} z_{i}, i \leq p$, the following are satisfied:

(1) $\left\{t_{i}: i \leq p\right\}$ is a maximal $S_{n_{j}}$ set and

$$
\left\|\sum_{i=1}^{p} a_{i} z_{i}\right\| \geq c_{1} \delta_{j}\left\|\sum_{i=1}^{p} a_{i} e_{t_{i}}\right\|_{n_{j}}
$$

for every sequence $\left(a_{i}\right)_{i=1}^{p}$ in $\mathbb{R}^{+}$.

(2) For every sequence $\left(a_{i}\right)_{i=1}^{p}$ in $\mathbb{R}$ with $\sum_{i=1}^{p}\left|a_{i}\right| \leq 1$,

$$
\left\|\sum_{i=1}^{p} a_{i} z_{i}\right\| \leq c_{2}\left\|\sum_{i=1}^{p} a_{i} e_{t_{i}}\right\|_{C k_{j}}+c_{3} \delta_{j}^{2} .
$$

Then $X$ has no infinite unconditional sequence. If moreover, given block subspaces $Y, Z$ of $X$ and $j \in \mathbb{N}$, such a block basis $\left(z_{i}\right)_{i=1}^{p}$ can be found with the additional property that $z_{i} \in Y$ if $i$ is odd, while $z_{i} \in Z$ if $i$ is even, then $X$ is H.I.

Proof. Let $\left(u_{i}\right),\left(v_{i}\right)$ be infinite block bases of $\left(x_{i}\right)$, and denote by $U$ and $V$ the block subspaces they generate respectively. Let $j \in \mathbb{N}$. Set $P=\left\{p_{i}\right.$ : $i \in \mathbb{N}\}$ and $Q=\left\{q_{i}: i \in \mathbb{N}\right\}$, where $p_{i}=\min \operatorname{supp} u_{i}$ and $q_{i}=\min \operatorname{supp} v_{i}$. According to the comments following the definition of the repeated averages hierarchy in Section 2, we can find $i_{0} \in \mathbb{N}$ so that if $L \in[P \cup Q]$ and $\min L \geq \min \left\{p_{i_{0}}, q_{i_{0}}\right\}$, then $\left\|\left[n_{j}\right]_{1}^{L}\right\|_{k_{j}}<\delta_{j}^{2}$. Let $Y=\left[u_{i}: i \geq i_{0}\right]$ and $Z=\left[v_{i}: i \geq i_{0}\right]$.

Choose $z_{1}<\ldots<z_{p}$ with $z_{i} \in Y$ when $i$ is odd, while $z_{i} \in Z$ when $i$ is even, according to the hypothesis. There exists $L \in[P \cup Q]$ with $\min L \geq$ $\min \left\{p_{i_{0}}, q_{i_{0}}\right\}$ such that $\left\{t_{i}: i \leq p\right\}=\operatorname{supp}\left[n_{j}\right]_{1}^{L}$. Put $a_{i}=\left[n_{j}\right]_{1}^{L}\left(t_{i}\right)$ for $i \leq p$, 
and note that $\left(a_{i}\right)_{i=1}^{p}$ is non-increasing. We now have

$$
\left\|\sum_{i=1}^{p} a_{i} z_{i}\right\| \geq c_{1} \delta_{j}\left\|\sum_{i=1}^{p} a_{i} e_{t_{i}}\right\|_{n_{j}}=c_{1} \delta_{j} .
$$

On the other hand,

$$
\left\|\sum_{i=1}^{p}(-1)^{i} a_{i} z_{i}\right\| \leq c_{2}\left\|\sum_{i=1}^{p} a_{i} e_{t_{i}}\right\|_{k_{j}}+c_{3} \delta_{j}^{2}
$$

as $\left(a_{i}\right)_{i=1}^{p}$ is non-increasing (see the fact mentioned at the end of Section 2). Hence,

$$
\left\|\sum_{i=1}^{p}(-1)^{i} a_{i} z_{i}\right\| \leq\left(c_{2}+c_{3}\right) \delta_{j}^{2} \leq \frac{c_{2}+c_{3}}{c_{1}} \delta_{j}\left\|\sum_{i=1}^{p} a_{i} z_{i}\right\| .
$$

Set $y=\sum_{i \text { odd }} a_{i} z_{i}$ and $z=\sum_{i \text { even }} a_{i} z_{i}$. We have shown that there exist non-zero vectors $y \in U$ and $z \in V$ so that $\|y-z\| \leq\left(\left(c_{2}+c_{3}\right) / c_{1}\right) \delta_{j}\|y+z\|$. Since $j$ was arbitrary, $X$ is H.I. The proof of the "moreover" statement is now complete. The proof of the first assertion is contained in the preceding argument if we take $U=V$.

4. Mixed Tsirelson spaces. Let $\mathcal{M}$ be a set of finitely supported signed measures on $\mathbb{N}$ which satisfies the following:

(1) $e_{n}^{*} \in \mathcal{M}$ for all $n \in \mathbb{N}$, where $e_{n}^{*}$ denotes the point mass at $n$.

(2) $\mathcal{M}$ is symmetric, i.e., if $\mu \in \mathcal{M}$ then $-\mu \in \mathcal{M}$,

(3) $\mathcal{M}$ is pointwise bounded, that is, $\mu(\{n\}) \leq 1$ for every $\mu \in \mathcal{M}$,

(4) $\mathcal{M}$ is closed under restriction to initial segments, i.e., if $\mu \in \mathcal{M}$, then $\mu \mid\{1, \ldots, n\} \in \mathcal{M}$.

Then one can define a norm $\|\cdot\|_{\mathcal{M}}$ on $c_{00}$ in the following manner:

$$
\left\|\sum_{i=1}^{\infty} a_{i} e_{i}\right\|_{\mathcal{M}}=\sup \left\{\sum_{i=1}^{\infty} a_{i} \mu(\{i\}): \mu \in \mathcal{M}\right\}
$$

for every finitely supported scalar sequence $\left(a_{i}\right)$. Of course, $\left(e_{i}\right)$ is the natural basis of $c_{00}$. Letting $X_{\mathcal{M}}$ denote the completion of $\left(c_{00},\|\cdot\|_{\mathcal{M}}\right)$, we see that $\left(e_{n}\right)$ is a normalized, monotone basis for $X_{\mathcal{M}}$. If $\mu \mid J \in \mathcal{M}$ for every $\mu \in \mathcal{M}$ and $J \subset \mathbb{N}$, then $\left(e_{n}\right)$ is 1-unconditional and bimonotone.

The main result of this section is

Theorem 4.1. Suppose $N$ is $M$-good. There exists a set $\mathcal{M}$ of finitely supported signed measures on $\mathbb{N}$ satisfying conditions (1)-(4) above and such that:

(1) $\left(e_{n}\right)$ is an 1-unconditional, shrinking, bimonotone basis for $X_{\mathcal{M}}$. 
(2) $X_{\mathcal{M}}$ has the $(6, N, P, \mathbf{a})$ distortion property, where $P=\left(f_{i}^{N}+2\right)$ and $\mathbf{a}=\left(1 / m_{i}\right)$.

We first give the construction of $\mathcal{M}$ and prove a number of lemmas necessary for the proof of Theorem 4.1.

Construction of $\mathcal{M}$. Given $M=\left(m_{i}\right), N=\left(n_{i}\right)$ with $N$ being $M$-good, we construct a set $\mathcal{M}$ of signed measures on $\mathbb{N}$ in the following manner: Let

$$
\begin{aligned}
\mathcal{D}=\left\{\left(t_{1}, \ldots, t_{3 n}\right): n \in \mathbb{N}, t_{3 i-2}\right. & \in M(i<n), t_{3 n-2}=0, \\
& \left.t_{3 i-1} \in[\mathbb{N}]<\infty \backslash\{\emptyset\}, t_{3 i} \in\{-1,1\}(i \leq n)\right\} .
\end{aligned}
$$

Given $F \in \mathcal{D}^{<\infty}, F \neq \emptyset$, we let $\mathcal{T}_{F}$ denote the set of all tuples of length divisible by 3 which are initial segments of elements of $F$. We can partially order the elements of $\mathcal{T}_{F}$ by initial segment inclusion; thus $\mathcal{T}_{F}$ becomes a finite tree with terminal nodes precisely the members of $F$. Given $\alpha \in \mathcal{T}_{F}$, every entry of $\alpha$ which belongs to $M$ is called an $M$-entry of $\alpha$. We shall denote the last three entries of $\alpha$ by $m_{\alpha}, I_{\alpha}$ and $\varepsilon_{\alpha}$ respectively. In case $m_{\alpha}=m_{i}$ for some $i \in \mathbb{N}$, we set $n_{\alpha}=n_{i}$. A rooted tree $\mathcal{T}=\mathcal{T}_{F}$ (a tree is rooted if it has a unique root) is said to be appropriate provided the following properties hold:

(1) If $\alpha \in \mathcal{T}$ is terminal, then $I_{\alpha}=\left\{p_{\alpha}\right\}$ for some $p_{\alpha} \in \mathbb{N}$.

(2) If $\alpha \in \mathcal{T}$ is non-terminal, then $\left(I_{\beta}\right)_{\beta \in D_{\alpha}}$ is $S_{n_{\alpha}}$-admissible (recall that if $m_{\alpha}=m_{j}$ for some $j \in \mathbb{N}$, then $n_{\alpha}=n_{j}$ ) and $I_{\alpha}=\bigcup_{\beta \in D_{\alpha}} I_{\beta}$. Here $D_{\alpha}$ stands for the set of immediate successors of $\alpha$ in $\mathcal{T}$.

We set

$$
\mathcal{G}=\{\mathcal{T}: \mathcal{T} \text { is an appropriate tree }\}
$$

We make the convention that the empty tree belongs to $\mathcal{G}$.

Notation. Let $\mathcal{T} \in \mathcal{G}$ and $\alpha \in \mathcal{T}$.

(1) $\alpha^{-}$stands for the predecessor of $\alpha$ in $\mathcal{T}$. In case $\alpha$ is the root of $\mathcal{T}$ we put $\alpha^{-}=\emptyset$.

(2) $|\alpha|$ is the length of $\alpha$. Thus, $|\alpha|=3 n$ if $\alpha=\left(t_{1}, \ldots, t_{3 n}\right)$. We define $o(\mathcal{T})=\max \{|\beta|: \beta \in \mathcal{T}\}$, the height of the tree $\mathcal{T}$.

(3) $m(\alpha)=\prod_{\beta<\alpha} m_{\beta}$ if $|\alpha|>3$, while $m(\alpha)=1$ if $|\alpha|=3$.

(4) $n(\alpha)=\sum_{\beta<\alpha} n_{\beta}$ if $|\alpha|>3$, while $n(\alpha)=0$ if $|\alpha|=3$.

Given $\mathcal{T} \in \mathcal{G}$, set

$$
\mu_{\mathcal{T}}=\sum_{\alpha \in \max \mathcal{T}} m(\alpha)^{-1} \varepsilon(\alpha) \varepsilon_{\alpha} e_{p_{\alpha}}^{*},
$$

where $\max \mathcal{T}$ is the set of terminal nodes of $\mathcal{T}$ and $I_{\alpha}=\left\{p_{\alpha}\right\}$ for $\alpha \in \max \mathcal{T}$. We have also set $\varepsilon(\alpha)=\prod_{\beta<\alpha} \varepsilon_{\alpha}$ for $\alpha \in \mathcal{T}$. We make the convention $\varepsilon(\alpha)=1$ if $|\alpha|=3$. We also set $\mu_{\emptyset}=0$. Of course, $\mu_{\mathcal{T}}$ is a finitely supported 
signed measure on $\mathbb{N}$ whose support is equal to $I_{\alpha_{0}}$, where $\alpha_{0}$ is the root of $\mathcal{T}$. Observe that $\left|\mu_{\mathcal{T}}(\{n\})\right| \leq 1$ for all $n \in \mathbb{N}$.

We finally set $\mathcal{M}=\left\{\mu_{\mathcal{T}}: \mathcal{T} \in \mathcal{G}\right\}$. Clearly, $e_{n}^{*} \in \mathcal{M}$ as $\{(0,\{n\}, 1)\} \in \mathcal{G}$. We introduce some more notation in order to investigate properties of the set $\mathcal{M}$.

Notation. Let $\mathcal{T} \in \mathcal{G}$ and let $\alpha_{0}$ denote its root.

(1) Given $\alpha \in \mathcal{T}$ set $\mathcal{T}_{\alpha}=\left\{\beta \backslash \alpha^{-}: \beta \in \mathcal{T}, \alpha \leq \beta\right\}$. Clearly, $\mathcal{T}_{\alpha} \in \mathcal{G}$.

(2) We let $w(\mathcal{T})=1$ if $|\mathcal{T}|=1$. In case $m_{\alpha_{0}} \in M$, we set $w(\mathcal{T})=m_{\alpha_{0}}$.

(3) Let $J \subset \mathbb{N}$. We let $\mathcal{T} \mid J$ denote the tree resulting from $\mathcal{T}$ by keeping only those $\alpha \in \mathcal{T}$ for which $I_{\alpha} \cap J \neq \emptyset$ and replacing $I_{\alpha}$ by $I_{\alpha} \cap J$. It is easy to see that $\mathcal{T} \mid J \in \mathcal{G}$.

(4) We let $-\mathcal{T}$ denote the tree resulting from $\mathcal{T}$ by changing $\varepsilon_{\alpha_{0}}$ to $-\varepsilon_{\alpha_{0}}$. Clearly, $-\mathcal{T} \in \mathcal{G}$ and moreover $\mu_{-\mathcal{T}}=-\mu_{\mathcal{T}}$.

REMARK 4.2. Let $\mathcal{T} \in \mathcal{G}$.

(1) If $J \subset \mathbb{N}$, then $\mu_{\mathcal{T} \mid J}=\mu_{\mathcal{T}} \mid J$.

(2) If $\alpha \in \mathcal{T}$ then $m(\alpha) \varepsilon(\alpha) \mu_{\mathcal{T}} \mid I_{\alpha}=\mu_{\mathcal{T}_{\alpha}}$.

REMARK 4.3. Suppose $\mathcal{T}_{i} \in \mathcal{G}, i \leq n$. Let $\alpha_{i}$ be the root of $\mathcal{T}_{i}$. We shall say that $\left\{\mathcal{T}_{i}: i \leq n\right\}$ is $S_{\xi^{-}}$admissible, $\xi<\omega$, if $\left\{I_{\alpha_{i}}: i \leq n\right\}$ is. We shall also write $\mathcal{T}_{1}<\ldots<\mathcal{T}_{n}$ if $I_{\alpha_{1}}<\ldots<I_{\alpha_{n}}$. It is not hard to see, using the second part of the preceding remark, that if $\mathcal{T}_{1}<\ldots<\mathcal{T}_{n}$ is $S_{n_{j}}$-admissible then $\left(\sum_{i=1}^{n} \mu_{\mathcal{T}_{i}}\right) / m_{j} \in \mathcal{M}$.

It follows by our preceding remarks that $\mathcal{M}$ is pointwise bounded, symmetric and closed under restriction to subsets of $\mathbb{N}$. Hence $\left(e_{n}\right)$ is an 1unconditional, bimonotone basis for $X_{\mathcal{M}}$. It is not hard to check that $X_{\mathcal{M}}$ is isometric to $T\left(1 / m_{i}, S_{n_{i}}\right)_{i=1}^{\infty}$. We also see from our preceding remarks that if $\left(x_{i}\right)_{i=1}^{k}$ is an $S_{n_{j}}$-admissible block basis of $\left(e_{n}\right)$ then $\left\|\sum_{i=1}^{k} x_{i}\right\| \geq$ $\left(1 / m_{j}\right) \sum_{i=1}^{k}\left\|x_{i}\right\|$. Hence, $\left(e_{n}\right)$ is a normalized basis for $X_{\mathcal{M}}$ with property $\left(1 / m_{j}\right)-\ell_{1}^{n_{j}}$ for all $j \in \mathbb{N}$. It follows that $\left(e_{n}\right)$ is boundedly complete. Let now $\nu$ be a $w^{*}$-cluster point of $\mathcal{M}$. Using the reflexivity argument of [4] (cf. also [30]), one finds that for every $\varepsilon>0$ there exists $k \in \mathbb{N}$ such that $\left\|\nu \mid\left[e_{i}: i \geq k\right]\right\|<\varepsilon$. It follows that $\left(e_{n}\right)$ is shrinking and thus $X_{\mathcal{M}}$ is reflexive.

REMARK 4.4. Suppose $\left(u_{n}\right)$ is a normalized block basis of $\left(e_{n}\right)$ and $u$ an $\left(\varepsilon, n_{j}\right)$ average of $\left(u_{n}\right)$. Then $1 / m_{j} \leq\|u\| \leq 1$.

LemMA 4.5. Let $\mathcal{T} \in \mathcal{G}$. Let $F$ be a subset of $\mathcal{T}$ consisting of pairwise incomparable nodes. Then $\left\{I_{\alpha}: \alpha \in F\right\}$ is $S_{p}$-admissible, where $p=$ $\max \{n(\alpha): \alpha \in F\}$.

Proof. By induction on $o(\mathcal{T})$. If $o(\mathcal{T})=3$ the assertion is trivial. Assuming it is true when $o(\mathcal{T})<3 k, k>1$, let $\mathcal{T} \in \mathcal{G}$ have $o(\mathcal{T})=3 k$. If $|F|=1$ 
there is nothing to prove. So assume $|F| \geq 2$. Let $\alpha_{0}$ be the root of $\mathcal{T}$ and let $w(\mathcal{T})=m_{i}$ for some $i \in \mathbb{N}$. We denote by $D$ the set of immediate successors of $\alpha_{0}$ in $\mathcal{T}$. Given $\alpha \in D$ let $F_{\alpha}=\{\beta \in F: \alpha \leq \beta\}$. Because $o\left(\mathcal{T}_{\alpha}\right) \leq 3 k-3$ we can apply the induction hypothesis to $\mathcal{T}_{\alpha}$ and the set $\left\{\beta \backslash \alpha^{-}: \beta \in F_{\alpha}\right\}$ to deduce that the collection $\left\{I_{\beta}: \beta \in F_{\alpha}\right\}$ is $S_{p_{1}}$-admissible, where $p_{1}=$ $\max \left\{n\left(\beta \backslash \alpha^{-}\right): \beta \in F_{\alpha}\right\}$. Since $n\left(\beta \backslash \alpha^{-}\right)=n(\beta)-n(\alpha)$ and $n(\alpha)=n_{i}$ whenever $\alpha \in D$, we conclude that $\left\{I_{\beta}: \beta \in F_{\alpha}\right\}$ is $S_{p-n_{i}}$-admissible, for every $\alpha \in D$. But also $\left\{I_{\alpha}: \alpha \in D\right\}$ is $S_{n_{i}}$-admissible, whence $\left\{I_{\alpha}: \alpha \in F\right\}$ is $S_{p}$-admissible.

To simplify our notation, we set $f_{j}=f_{j}^{N}$. We make the following observation: Let $\mathcal{T} \in \mathcal{G}$ and $\alpha \in \mathcal{T}$. Assume that $m(\alpha)<m_{j}^{3}$ and all $M$-entries of $\alpha^{-}$are smaller than $m_{j}$. Then $n(\alpha) \leq f_{j}$. Our next lemma will be crucial for the proof of the main result.

Lemma 4.6 (Decomposition Lemma). Let $\mathcal{T}_{0} \in \mathcal{G}$. Let $j \in \mathbb{N}$ be such that $w\left(\mathcal{T}_{0}\right)<m_{j}$. Then there exist an $S_{f_{j}}$-admissible subset $\mathcal{G}_{0}$ of $\mathcal{G}$ and $a$ scalar sequence $\left(\lambda_{\mathcal{T}}\right)_{\mathcal{T} \in \mathcal{G}_{0}}$ in $[-1,1]$ so that:

(1) $\mu_{\mathcal{T}_{0}}=\sum_{\mathcal{T} \in \mathcal{G}_{0}} \lambda_{\mathcal{T}} \mu_{\mathcal{T}}$.

(2) For each $\mathcal{T} \in \mathcal{G}_{0}$, either $w(\mathcal{T})=1$ (thus $\mu_{\mathcal{T}}= \pm e_{\mathcal{T}(p)}^{*}$ for some $\mathcal{T}(p) \in \mathbb{N})$, or $w(\mathcal{T}) \geq m_{j}$, or $\left|\lambda_{\mathcal{T}}\right| \leq 1 / m_{j}^{2}$.

Proof. Let $\mathfrak{B}$ denote the set of all branches of $\mathcal{T}_{0}$ (a branch is a maximal well ordered subset of $\mathcal{T}_{0}$ ). If $w\left(\mathcal{T}_{0}\right)=1$ the assertion is trivial. So assume that $w\left(\mathcal{T}_{0}\right)=m_{i_{0}}$ for some $i_{0}<j$. Given $b \in \mathfrak{B}$ set

$$
\alpha^{1}(b)=\max \left\{\beta \in b: m(\beta)<m_{j}^{2} \text { and if } m_{i} \in \beta^{-} \text {then } i<j\right\} .
$$

Note that $\alpha^{1}(b)$ is well defined and $\left(m_{i_{0}}, I, \varepsilon\right)<\alpha^{1}(b)$ since $i_{0}<j\left(\left(m_{i_{0}}, I, \varepsilon\right)\right.$ being the root of $\mathcal{T}_{0}$ ).

Let us say that $b \in \mathfrak{B}$ is of type 1 if $\alpha^{1}(b)$ is terminal in $\mathcal{T}_{0}$. If $b$ is not of type 1 then it is of type 2 (resp. 3) if the last $M$-entry of $\alpha^{1}(b)$ is greater than or equal to (resp. smaller than) $m_{j}$. We then denote by $\alpha^{2}(b)$ the immediate successor of $\alpha^{1}(b)$ in $b$.

We let $A_{1}=\left\{\alpha^{1}(b): b \in \mathfrak{B}\right.$ of type 1$\}, A_{2}=\left\{\alpha^{1}(b): b \in \mathfrak{B}\right.$ of type 2$\}$ and $A_{3}=\left\{\alpha^{2}(b): b \in \mathfrak{B}\right.$ of type 3$\}$. Observe that:

(1) If $\alpha \in A_{3}$ then all $M$-entries of $\alpha^{-}$are smaller than $m_{j}, m\left(\alpha^{-}\right)<m_{j}^{2}$, yet $m_{j}^{2} \leq m(\alpha)<m_{j}^{3}$.

(2) If $\alpha \in A_{2}$, then $\alpha$ is non-terminal, all $M$-entries in $\alpha^{-}$are smaller than $m_{j}$, the last $M$-entry of $\alpha$ is greater than or equal to $m_{j}$ and $m(\alpha)<m_{j}^{2}$.

(3) If $\alpha \in A_{1}$ then $\alpha$ is terminal, all $M$-entries in $\alpha^{-}$are smaller than $m_{j}$ and $m(\alpha)<m_{j}^{2}$. 
It is not hard to check now that $A=\bigcup_{t=1}^{3} A_{t}$ consists of pairwise incomparable nodes of $\mathcal{T}_{0}$ and hence $\left\{I_{\alpha}: \alpha \in A\right\}$ consists of successive subsets of $\mathbb{N}$. Moreover, $I=\bigcup\left\{I_{\alpha}: \alpha \in A\right\}$. Because $m(\alpha)<m_{j}^{3}$ and all $M$-entries of $\alpha^{-}$are smaller than $m_{j}$ whenever $\alpha \in A$, we obtain $n(\alpha) \leq f_{j}$ for all $\alpha \in A$. Lemma 4.5 now shows that $\left\{I_{\alpha}: \alpha \in A\right\}$ is $S_{f_{j}}$-admissible. Finally, we let $\mathcal{G}_{0}=\left\{\left(\mathcal{T}_{0}\right)_{\alpha}: \alpha \in A\right\}$. Since $m(\alpha) \varepsilon(\alpha) \mu_{\mathcal{T}_{0}} \mid I_{\alpha}=\mu_{\left(\mathcal{T}_{0}\right)_{\alpha}}$ for all $\alpha \in \mathcal{T}$, we set $\lambda_{\left(\mathcal{T}_{0}\right)_{\alpha}}=1 /(m(\alpha) \varepsilon(\alpha))$ for $\alpha \in A$. We can easily verify that the desired properties hold.

In what follows, we shall be using a variety of block bases of $\left(e_{n}\right)$. The support of each of them will always be taken with respect to $\left(e_{n}\right)$.

Lemma 4.7. Let $\left(u_{n}\right)$ be a normalized block basis of $\left(e_{n}\right)$. Let $j \in \mathbb{N}$, $j \geq 2$ and let $u$ be a generic $\left(\varepsilon, f_{j}+1\right)$ average of $\left(u_{n}\right)$ with $\varepsilon<1 /\left(2 m_{j}\right)$. Let $i<j$ and let $\mathcal{T}_{1}<\ldots<\mathcal{T}_{t}$ in $\mathcal{G}$ be $S_{n_{i}}$-admissible. Then $\sum_{k=1}^{t} \mu_{\mathcal{T}_{k}}(u) \leq 2$. In particular, $\mu_{\mathcal{T}}(u) \leq 2 / w(\mathcal{T})$ if $w(\mathcal{T})<m_{j}$.

Proof. Observe that $\left(1 / m_{j}\right) \sum_{k=1}^{t} \mu_{\mathcal{T}_{k}} \in \mathcal{M}$ and hence $\sum_{k=1}^{t} \mu_{\mathcal{T}_{k}}\left(u_{n}\right)$ $\leq m_{j}$ for all $n \in \mathbb{N}$. Let $P=\left(p_{n}\right)$, where $p_{n}=\min \operatorname{supp} u_{n}$. Set $\xi=$ $f_{j}+1$ and suppose that $u=\sum_{n=1}^{\infty} \xi_{1}^{R}\left(p_{n}\right) u_{n}$ for some $R \in[P]$. Let $E^{*}$ denote the collection of the ranges of the $\mu_{\mathcal{T}_{k}}$ 's, and $D$ the collection of those $u_{n}$ 's whose support intersects at least one member of $E^{*}$. Put $I_{r}=$ $\left\{n \in \mathbb{N}: u_{n} \in D\left(E^{*}, r\right)\right\}, r=1,2$ (see the notation introduced in Section 2). Because $D\left(E^{*}, 2\right)$ is $2 S_{n_{i}}$-admissible (see Remark 2.2$)$ and $n_{i} \leq f_{j}$, we obtain $\sum_{k=1}^{t} \mu_{\mathcal{T}_{k}}\left(\sum_{n \in I_{2}} \xi_{1}^{R}\left(p_{n}\right) u_{n}\right) \leq m_{j} 2 \varepsilon$. On the other hand we clearly have $\sum_{k=1}^{t} \mu_{\mathcal{T}_{k}}\left(\sum_{n \in I_{1}} \xi_{1}^{R}\left(p_{n}\right) u_{n}\right) \leq 1$. Thus, $\sum_{k=1}^{t} \mu_{\mathcal{T}_{k}}(u) \leq 2$.

LEMMA 4.8. Let $\left(u_{n}\right)$ be a normalized block basis of $\left(e_{n}\right)$. Let $\varepsilon>0$ and $j \in \mathbb{N}$. Then there exists a smoothly normalized (Definition 2.1) $\left(\varepsilon, f_{j}+1\right)$ average of $\left(u_{n}\right)$.

Proof. Let $P=\left(p_{n}\right)$, where $p_{n}=\min \operatorname{supp} u_{n}$ for $n \in \mathbb{N}$. We can assume without loss of generality that $\left\|\xi_{1}^{R}\right\|_{\xi-1}<\varepsilon$ for every $R \in[P]$, where $\xi=$ $f_{j}+1$. We are going to show that there exists a normalized block basis of $\left(u_{n}\right)$ admitting a generic $(\varepsilon, \xi)$ average of norm at least $1 / 2$. Suppose this were false. Then it is easy to construct, for every $1 \leq r \leq l_{j}$, a block basis $\left(u_{i}^{r}\right)$ of $\left(u_{i}\right)$ so that letting $p_{i}^{r}=\min \operatorname{supp} u_{i}^{r}$ and $P_{r}=\left(p_{i}^{r}\right)$ the following are satisfied:

(1) $\left(u_{i}^{r}\right)$ is a block basis of $\left(u_{i}^{r-1}\right)\left(u_{i}^{0}=u_{i}\right)$.

(2) $u_{i}^{r}=\sum_{n=1}^{\infty} \xi_{i}^{P_{r-1}}\left(p_{n}^{r-1}\right) u_{n}^{r-1} /\left\|u_{n}^{r-1}\right\|$ for all $i \in \mathbb{N}\left(p_{n}^{0}=p_{n}\right)$.

(3) $\left\|u_{i}^{r}\right\|<1 / 2$ for all $i \in \mathbb{N}$.

(4) For every $i \in \mathbb{N}$, if $u_{i}^{r}=\sum_{n \in F_{i}^{r}} a_{n} u_{n}$ with $a_{n}>0$ for $n \in F_{i}^{r}$, then $\sum_{n \in F_{i}^{r}} a_{n} \geq 2^{r-1}$ and $\left(u_{n}\right)_{n \in F_{i}^{r}}$ is $S_{\xi r^{-}}$-admissible. 
The construction is easily done by induction. Taking $r=l_{j}$ we see from (3) that $\left\|u_{i}^{l_{j}}\right\|<1 / 2$. On the other hand, (4) implies that $\left\|u_{i}^{l_{j}}\right\| \geq 2^{l_{j}-1} / m_{j}$ as $\xi l_{j}<n_{j}$. Thus, $m_{j}>2^{l_{j}}$, contradicting the choice of $l_{j}$.

Our next lemma implies that $X_{\mathcal{M}}$ has the $(6, N, F, \mathbf{a})$ distortion property where $F=\left(f_{i}+2\right)$ and $\mathbf{a}=\left(1 / m_{i}\right)$.

Lemma 4.9. Let $\left(u_{j}\right)$ be a normalized block basis of $\left(e_{j}\right)$. Suppose that $\left(y_{j}\right)$ is a block basis of $\left(u_{j}\right)$ so that $y_{j}$ is a smoothly normalized $\left(\varepsilon_{j}, f_{j}+1\right)$ average of $\left(u_{j}\right)$ with $\varepsilon_{j}<1 /\left(2 m_{j}\right)$ (Definition 2.1). Given $j_{0} \in \mathbb{N}$ and $J_{0} \in[\mathbb{N}]$, there exists $J \in\left[J_{0}\right]$ such that $j_{0}<\min J$ and for every $\mathcal{T} \in \mathcal{G}$, $D_{\mathcal{T}}=\left\{y_{j}: j \in J,\left|\mu_{\mathcal{T}}\left(y_{j}\right)\right| \geq 5 / m_{j_{0}}\right\}$ is $S_{f_{j_{0}}+1}$-admissible.

Proof. Note first that Lemma 4.8 guarantees the existence of the block basis $\left(y_{j}\right)$. Let $P=\left(p_{j}\right)_{j \in J_{0}}$, where $p_{j}=\min \operatorname{supp} y_{j}$. By passing to a subsequence of $\left(y_{j}\right)_{j \in J_{0}}$ if necessary, we can assume that the union of any four $S_{f_{j_{0}}}$ subsets of $P$ belongs to $S_{f_{j_{0}}+1}$. Choose $J=\left(j_{i}\right) \in\left[J_{0}\right]$ such that $j_{0}<j_{1}$ and $\left\|y_{j_{i}}\right\|_{\ell_{1}}<m_{j_{i+1}} / m_{j_{i}}$ for every $i \geq 2$ (if $v=\sum_{i=1}^{n} a_{i} e_{i}$, then $\left.\|v\|_{\ell_{1}}=\sum_{i=1}^{n}\left|a_{i}\right|\right)$.

Let $\mathcal{T}_{0} \in \mathcal{G}$. Suppose first that $w\left(\mathcal{T}_{0}\right) \geq m_{j_{0}}$. We show that in this case $\left|D_{\mathcal{T}_{0}}\right| \leq 1$. Indeed, suppose first that $w\left(\mathcal{T}_{0}\right)<m_{j_{1}}$. Lemma 4.7 shows that $\left|\mu_{\mathcal{T}_{0}}\left(y_{j}\right)\right| \leq 4 / m_{j_{0}}$ for all $j \in J$, whence $D_{\mathcal{T}_{0}}=\emptyset$.

If $w\left(\mathcal{T}_{0}\right) \geq m_{j_{1}}$ choose $s \geq 2$ so that $m_{j_{s-1}} \leq w\left(\mathcal{T}_{0}\right)<m_{j_{s}}$. Observe that if $1 \leq i<s-1$ then

$$
\left|\mu_{\mathcal{T}_{0}}\left(y_{j_{i}}\right)\right| \leq \frac{1}{w\left(\mathcal{T}_{0}\right)} \cdot\left\|y_{j_{i}}\right\|_{\ell_{1}}<\frac{1}{w\left(\mathcal{T}_{0}\right)} \cdot \frac{m_{j_{s-1}}}{m_{j_{i}}}<\frac{1}{m_{j_{0}}} .
$$

When $i \geq s$, Lemma 4.7 yields $\left|\mu_{\mathcal{T}_{0}}\left(y_{j_{i}}\right)\right| \leq 4 / w\left(\mathcal{T}_{0}\right)<4 / m_{j_{0}}$. Hence $D_{\mathcal{T}_{0}} \subset$ $\left\{y_{j_{s-1}}\right\}$ and so our claim holds.

The final case to consider is that of $w\left(\mathcal{T}_{0}\right)<m_{j_{0}}$. Clearly, $D_{\mathcal{T}_{0}}=\emptyset$ if $w\left(\mathcal{T}_{0}\right)=1$. We employ the decomposition Lemma 4.6 to find an $S_{f_{j_{0}}}$ admissible subset $\mathcal{G}_{0}$ of $\mathcal{G}$ and scalars $\left(\lambda_{\mathcal{T}}\right)_{\mathcal{T} \in \mathcal{G}_{0}}$ satisfying the conclusion of Lemma 4.6. Let $E^{*}$ denote the collection of the ranges of the $\mu_{\mathcal{T}}$ 's $\left(\mathcal{T} \in \mathcal{G}_{0}\right)$. Our previous work combined with Remark 2.2 implies that $D_{\mathcal{T}_{0}}\left(E^{*}, 1\right)$ is $2 S_{f_{0}}$-admissible. But also $D_{\mathcal{T}_{0}}\left(E^{*}, 2\right)$ is $2 S_{f_{j_{0}}}$-admissible (again by Remark 2.2 ) since $\mathcal{G}_{0}$ is $S_{f_{j_{0}}}$-admissible. It follows that $D_{\mathcal{T}_{0}}$ is $S_{f_{j_{0}}+1^{-}}$-admissible.

Proof of Theorem 4.1. Let $\left(u_{n}\right)$ be a normalized block basis of $\left(e_{n}\right)$. Let $j_{0} \in \mathbb{N}$ and choose a block basis $\left(y_{j}\right)_{j \in J}$ of $\left(u_{n}\right)$ satisfying the conclusion of Lemma 4.9. Set $\xi=f_{j_{0}}+2$. We claim that if $\left(z_{n}\right)$ is a subsequence of $\left(y_{j}\right)_{j \in J}$ which is a $\delta$ - $\ell_{1}^{\xi}$ spreading model, then $\delta \leq 5 / m_{j_{0}}$. Indeed, let $p_{n}=\min \operatorname{supp} z_{n}$ and $P=\left(p_{n}\right)$. According to Corollary 3.4 of [2] (cf. also Corollary 3.3 of [12]), there exists $Q=\left(q_{n}\right) \in[P]$ such that if $F \in S_{\xi} \cap[Q]^{<\infty}$, then there exists $G \in S_{\xi}$ with $F \backslash\{\min F\}=\left(p_{n}\right)_{n \in G}$. Suppose that $q_{n}=p_{k_{n}}$ for all $n \in \mathbb{N}$. It follows that if $\left(q_{n}\right)_{n \in H} \in S_{\xi}$, then 
$\left(k_{n}\right)_{n \in H \backslash\{\min H\}} \in S_{\xi}$. We deduce that if $\left(z_{k_{n}}\right)_{n \in H}$ is $S_{\xi}$-admissible, then $\left(k_{n}\right)_{n \in H \backslash\{\min H\}} \in S_{\xi}$ and thus $\left\|\sum_{n \in H} a_{n} z_{k_{n}}\right\| \geq \delta(1-\varepsilon)-\varepsilon$ for every choice of non-negative scalars $\left(a_{n}\right)_{n \in H}$ such that $a_{n}<\varepsilon$ for all $n \in H$ and $\sum_{n \in H} a_{n}=1$.

Let now $u=\sum_{n \in H} a_{n} z_{k_{n}}$ be a generic $(\varepsilon, \xi)$ average of $\left(z_{k_{n}}\right)$. Then

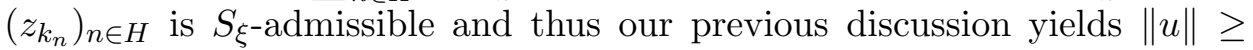
$\delta(1-\varepsilon)-\varepsilon$. On the other hand, Lemma 4.9 implies that $\|u\| \leq 5 / m_{j_{0}}+\varepsilon$, since $f_{j_{0}}+1<\xi$. Our estimates now yield $\delta \leq 5 / m_{j_{0}}$, as $\varepsilon>0$ was arbitrary. We conclude that $\tau\left(\left(u_{n}\right), 6 / m_{j_{0}}\right)<f_{j_{0}}+2$, as desired.

Terminology. Let $j_{0}$ and $\left(y_{j}\right)_{j \in J}$ be chosen as in the proof of Lemma 4.9. Every normalized $\left(\varepsilon, n_{j_{0}}\right)$ average $u$ of $\left(u_{j}\right)_{j=1}^{\infty}$ of the form $u=v /\|v\|$, where $v$ is a generic $\left(\varepsilon, n_{j_{0}}\right)$ average of $\left(y_{j}\right)_{j \in J}$, will be called a normalized $\left(\varepsilon, n_{j_{0}}\right)$ average of $\left(u_{j}\right)_{j=1}^{\infty}$ resulting from Lemma 4.9 . Note that Lemmas 4.8 and 4.9 guarantee the existence of such averages for every block basis $\left(u_{j}\right)_{j=1}^{\infty}$.

COROLlarY 4.10. Let $j_{0}$ and $\left(y_{j}\right)_{j \in J}$ be chosen as in the proof of Lemma 4.9. Then for every $\mathcal{T}_{0} \in \mathcal{G}$ with $w\left(\mathcal{T}_{0}\right) \neq m_{j_{0}}$, the block basis $Q_{\mathcal{T}_{0}}=$ $\left\{y_{j}: j \in J,\left|\mu_{\mathcal{T}_{0}}\left(y_{j}\right)\right| \geq 5 /\left(m_{j_{0}} m_{e}\right)\right\}$ is $S_{f_{j_{0}}+1^{-}}$admissible, where $m_{e}=$ $\min \left\{m_{j_{0}}, w\left(\mathcal{T}_{0}\right)\right\}$.

Proof. We may assume that $w\left(\mathcal{T}_{0}\right)>1$ or else the assertion is trivial. Suppose first that $w\left(\mathcal{T}_{0}\right)>m_{j_{0}}$. Because $m_{i}^{2}<m_{i+1}$, the argument in the proof of Lemma 4.9 shows that $\left|Q_{\mathcal{T}_{0}}\right| \leq 1$.

When $w\left(\mathcal{T}_{0}\right)<m_{j_{0}}$, we apply the decomposition Lemma 4.6 to find an $S_{f_{j_{0}}}$-admissible subset $\mathcal{G}_{0}$ of $\mathcal{G}$ and scalars $\left(\lambda_{\mathcal{T}}\right)_{\mathcal{T} \in \mathcal{G}_{0}}$ satisfying the conclusion of Lemma 4.6. Note that if $\mathcal{T} \in \mathcal{G}_{0}$ then $\left|\lambda_{\mathcal{T}}\right| \leq 1 / w\left(\mathcal{T}_{0}\right)$. Let $E^{*}$ denote the collection of the ranges of the $\mu_{\mathcal{T}}$ 's $\left(\mathcal{T} \in \mathcal{G}_{0}\right)$. Let $y_{j} \in Q_{\mathcal{T}_{0}}\left(E^{*}, 1\right)$. Then there exists a unique $\mathcal{T}_{j} \in \mathcal{G}_{0}$ such that $\left|\lambda_{\mathcal{T}_{j}} \mu_{\mathcal{T}_{j}}\left(y_{j}\right)\right| \geq 5 /\left(m_{j_{0}} w\left(\mathcal{T}_{0}\right)\right)$. Since $w\left(\mathcal{T}_{0}\right)<m_{j_{0}}$, we must have $w\left(\mathcal{T}_{j}\right) \geq m_{j_{0}}$ and thus $\left|\mu_{\mathcal{T}_{j}}\left(y_{j}\right)\right| \geq 5 / m_{j_{0}}$. Hence, $y_{j} \in D_{\mathcal{T}_{j}}$ (see the notation in Lemma 4.9). Because $\left|D_{\mathcal{T}_{j}}\right| \leq 1$, by the proof of Lemma 4.9 , we get $D_{\mathcal{T}_{j}}=\left\{y_{j}\right\}$.

We now define a map $\phi: Q_{\mathcal{T}_{0}}\left(E^{*}, 1\right) \rightarrow\left\{\mathcal{T} \in \mathcal{G}_{0}: w(\mathcal{T}) \geq m_{j_{0}}\right\}$ by setting $\phi\left(y_{j}\right)=\mathcal{T}_{j}$. Then $\phi$ is injective as $D_{\mathcal{T}_{j}}=\left\{y_{j}\right\}$. It follows that $Q_{\mathcal{T}_{0}}\left(E^{*}, 1\right)$ is $2 S_{f_{j_{0}}}$-admissible since $\mathcal{G}_{0}$ is $S_{f_{j_{0}}}$-admissible. But also $Q_{\mathcal{T}_{0}}\left(E^{*}, 2\right)$ is $2 S_{f_{j_{0}}}$ admissible and hence $Q_{\mathcal{T}_{0}}$ is $S_{f_{j_{0}}+1^{-}}$-admissible.

COROLlary 4.11. Let $u$ be a normalized $\left(\varepsilon, n_{j_{0}}\right)$ average of $\left(u_{j}\right)_{j=1}^{\infty}$ resulting from Lemma 4.9 with $\varepsilon \leq 1 /\left(12 m_{j_{0}}^{2}\right)$. Let $\mathcal{G}_{0}$ be an $S_{n_{i}}$-admissible subset of $\mathcal{G}$, for some $i<j_{0}$, such that $m_{j_{0}} \notin\left\{w(\mathcal{T}): \mathcal{T} \in \mathcal{G}_{0}\right\}$. Then $\left|\sum_{\mathcal{T} \in \mathcal{G}_{0}} \mu_{\mathcal{T}}(u)\right| \leq 7 / m_{e}$, where $m_{e}=\min \left\{w(\mathcal{T}): w(\mathcal{T})>1, \mathcal{T} \in \mathcal{G}_{0}\right\} \cup$ $\left\{m_{j_{0}}\right\}$. 
Proof. Set $\xi=n_{j_{0}}$. Let $p_{j}=\min \operatorname{supp} y_{j}$ for $j \in J$ and $P=\left\{p_{j}: j \in J\right\}$. There exists $R \in[P]$ so that $u=v /\|v\|$, where $v=\sum_{j \in J} \xi_{1}^{R}\left(p_{j}\right) y_{j}$ and $\left\|\xi_{1}^{R}\right\|_{n_{j_{0}}-1}<\varepsilon$. Note that $\|v\| \geq 1 / m_{j_{0}}$.

Suppose first that $w(\mathcal{T})>1$ for all $\mathcal{T} \in \mathcal{G}_{0}$. Let $Q=\left\{y_{j}: j \in J\right.$, $\left.\left|\sum_{\mathcal{T} \in \mathcal{G}_{0}} \mu_{\mathcal{T}}\left(y_{j}\right)\right| \geq 5 /\left(m_{j_{0}} m_{e}\right)\right\}$. Let $E^{*}$ denote the collection of the ranges of the $\mu_{\mathcal{T}}$ 's $\left(\mathcal{T} \in \mathcal{G}_{0}\right)$. Applying Corollary 4.10 and taking into account Remark 2.2, we find that $Q\left(E^{*}, 1\right)$ is $2 S_{f_{j_{0}}+1+n_{i}}$-admissible. But also $Q\left(E^{*}, 2\right)$

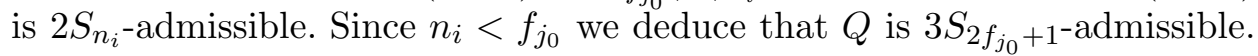
The assertion now follows from Lemma 4.7 and the fact that $2 f_{j_{0}}+1$ $<n_{j_{0}}$.

Next suppose that $w(\mathcal{T})=1$ for all $\mathcal{T} \in \mathcal{G}_{0}$. It is easy to see, using Lemma 4.7 , that in this case one obtains the estimate $\left|\sum_{\mathcal{T} \in \mathcal{G}_{0}} \mu_{\mathcal{T}}(u)\right| \leq 1 / m_{j_{0}}$. The result now follows by combining the previously discussed cases.

5. Hereditarily indecomposable spaces. This section is devoted to the proof of Theorem 3.5. Recall that $X$ is H.I. if and only if, for every pair of subspaces $Y, Z$ of $X$ and every $\varepsilon>0$, there exist non-zero vectors $y \in Y$ and $z \in Z$ so that $\|y-z\| \leq \varepsilon\|y+z\|$.

Let $M=\left(m_{i}\right) \in[\mathbb{N}]$ and let $N=\left(n_{i}\right) \in[\mathbb{N}]$ be $M$-good. Let $\mathcal{M}$ be the set of measures constructed in the previous section by using the sets $M$ and $N$. We shall choose $\mathcal{N} \subset \mathcal{M}$ so that the resulting space $X_{\mathcal{N}}$ is a reflexive H.I. space satisfying the conclusion of Theorem 3.5. We can find an injection

$$
\sigma:\left\{\left(\mathcal{T}_{1}<\ldots<\mathcal{T}_{n}\right): n \in \mathbb{N}, \mathcal{T}_{i} \in \mathcal{G}(i \leq n)\right\} \rightarrow\left\{m_{2 j}: j \in \mathbb{N}\right\}
$$

so that $\sigma\left(\mathcal{T}_{1}, \ldots, \mathcal{T}_{n}\right)>w\left(\mathcal{T}_{i}\right)$ for all $i \leq n$.

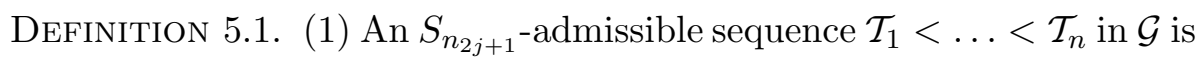
said to be $S_{n_{2 j+1}}$-dependent, $j \geq 0$, if $w\left(\mathcal{T}_{1}\right)=m_{2 j_{1}}$ for some $j_{1}>(2 j+1) / 2$, and $\sigma\left(\mathcal{T}_{1}, \ldots, \mathcal{T}_{i-1}\right)=w\left(\mathcal{T}_{i}\right)$ for all $2 \leq i \leq n$.

(2) Let $\mathcal{T}_{1}<\ldots<\mathcal{T}_{n}$ in $\mathcal{G}, j \geq 0$ and $\mathcal{G}_{0} \subset \mathcal{G}$. We shall say that $\mathcal{T}_{1}<\ldots<\mathcal{T}_{n}$ admits an $S_{n_{2 j+1}}$-dependent extension in $\mathcal{G}_{0}$ if there exist $l \in \mathbb{N}, k \in \mathbb{N} \cup\{0\}$ and an $S_{n_{2 j+1}}$-dependent sequence $\mathcal{R}_{1}<\ldots<\mathcal{R}_{n+k}$ in $\mathcal{G}_{0}$ so that $\mathcal{R}_{k+i} \mid[l, \infty)=\mathcal{T}_{i}$ for all $i \leq n$.

(3) A subset $\mathcal{G}_{0}$ of $\mathcal{G}$ is said to be self-dependent if the following condition is satisfied for every $\mathcal{T} \in \mathcal{G}_{0}$ : Let $\alpha \in \mathcal{T}$ be such that $m_{\alpha}=m_{2 j+1}$ for some $j \geq 0$. Let $D_{\alpha}$ denote the set of immediate successors of $\alpha$ in $\mathcal{T}$. Then $\left\{\mathcal{T}_{\beta}: \beta \in D_{\alpha}\right\}$ admits an $S_{n_{2 j+1}}$-dependent extension in $\mathcal{G}_{0}$.

Definition 5.2. We let $\mathfrak{D}$ denote the union of all non-empty, self-dependent, symmetric subsets of $\mathcal{G}$ closed under restriction to intervals. Recall that $\mathcal{G}_{0} \subset \mathcal{G}$ is symmetric if $-\mathcal{T} \in \mathcal{G}_{0}$ whenever $\mathcal{T} \in \mathcal{G}_{0}$. Moreover, $\mathcal{G}_{0}$ is closed under interval restrictions if $\mathcal{T} \mid J \in \mathcal{G}_{0}$ whenever $\mathcal{T} \in \mathcal{G}_{0}$ and $J$ is an interval. 
Of course $\mathfrak{D}$ is a maximal, under inclusion, subset of $\mathcal{G}$ with respect to the aforementioned properties. Set $\mathcal{N}=\left\{\mu_{\mathcal{T}}: \mathcal{T} \in \mathfrak{D}\right\}$. We will show that $X_{\mathcal{N}}$ is H.I.

REMARK 5.3. The maximality of $\mathfrak{D}$ implies the following:

(1) $e_{n}^{*} \in \mathcal{N}$ for all $n \in \mathbb{N}$.

(2) If $\mathcal{T} \in \mathfrak{D}$, then $\mathcal{T}_{\alpha} \in \mathfrak{D}$ for all $\alpha \in \mathcal{T}$ and so the decomposition Lemma 4.6 holds for $\mathfrak{D}$.

(3) If $\mathcal{T}_{1}<\ldots<\mathcal{T}_{k}$ in $\mathfrak{D}$ is $S_{n_{2 i}}$-admissible and $i \in \mathbb{N}$, then $\left(\mu_{\mathcal{T}_{1}}+\ldots\right.$ $\left.+\mu_{\mathcal{T}_{k}}\right) / m_{2 i} \in \mathcal{N}$.

(4) If $\mathcal{T}_{1}<\ldots<\mathcal{T}_{k}$ in $\mathfrak{D}$ is $S_{n_{2 i+1}}$-dependent and $i \in \mathbb{N}$, then $\left(\mu_{\mathcal{T}_{1}}+\ldots\right.$ $\left.+\mu_{\mathcal{T}_{k}}\right) / m_{2 i+1} \in \mathcal{N}$.

(5) Because of (3), all the results obtained in the previous section about $(\varepsilon, \xi)$ averages in $X_{\mathcal{M}}$, where $\xi$ is either $n_{j}$ or $f_{j}+1$ for some $j \in \mathbb{N}$, still hold in $X_{\mathcal{N}}$ provided $j$ is even.

Note that $X_{\mathcal{N}}$ is reflexive by the same argument that showed $X_{\mathcal{M}}$ was reflexive. Thus $\left(e_{i}\right)$ is a shrinking basis for $X_{\mathcal{N}}$.

Proof of Theorem 3.5. It follows from Theorem 4.1 and our preceding remarks that $X_{\mathcal{N}}$ has the $\left(6, N^{(2)}, F^{(2)}\right.$,a) distortion property. We show that $X_{\mathcal{N}}$ is H.I. This is accomplished through Theorem 3.6. Let $\left(u_{n}\right)$ and $\left(v_{n}\right)$ be normalized block bases of $\left(e_{n}\right)$ and let $j \in \mathbb{N}$. Set $P=\left(p_{n}\right)$ and $Q=\left(q_{n}\right)$, where $p_{n}=\min \operatorname{supp} u_{n}$ and $q_{n}=\min \operatorname{supp} v_{n}$ for all $n \in \mathbb{N}$. We can assume that the union of any seven $S_{f_{2 j+1}}$ subsets of $P \cup Q$ belongs to $S_{f_{2 j+1}+1}$. Successive applications of Corollary 4.11 yield a normalized block basis $g_{1}<\ldots<g_{p}$ of $\left(e_{n}\right), \mathcal{T}_{1}<\ldots<\mathcal{T}_{p}$ in $\mathfrak{D}$, and integers $j_{1}<\ldots<j_{p}$ with $2 j+1<j_{1}$, satisfying the following:

(1) $g_{i}$ is a normalized $\left(1 /\left(12 m_{2 j_{i}}^{2}\right), n_{2 j_{i}}\right)$ average of $\left(u_{n}\right)$ (resp. $\left.\left(v_{n}\right)\right)$ resulting from Lemma 4.9 when $i$ is odd (resp. even).

(2) $w\left(\mathcal{T}_{i}\right)=m_{2 j_{i}}, \operatorname{supp} \mu_{\mathcal{T}_{i}} \subset r\left(g_{i}\right)$ and $\mu_{\mathcal{T}_{i}}\left(g_{i}\right)>1 / 2$ for all $i \leq p$ (here we used the fact that $\left.m_{1}>7\right)$.

(3) $\sigma\left(\mathcal{T}_{1}, \ldots, \mathcal{T}_{i-1}\right)=w\left(\mathcal{T}_{i}\right)$ for all $2 \leq i \leq p$.

(4) $\left\{g_{i}: i \leq p\right\}$ is maximally $S_{n_{2 j+1}}$-admissible.

Put $\theta_{i}=\mu_{\mathcal{T}_{i}}\left(g_{i}\right)^{-1}, z_{i}=\theta_{i} g_{i}$, and note that $1 \leq \theta_{i}<2, i \leq p$. We show that $\left(z_{i}\right)_{i=1}^{p}$ satisfies conditions (1) and (2) of Theorem 3.6, with $\delta_{j}=1 / m_{2 j+1}$, " $n_{j} "=n_{2 j+1}$ and $k_{j}=f_{2 j+1}+1$. Condition (1) is immediate since $\mathcal{T}_{1}<$ $\ldots<\mathcal{T}_{p}$ is $S_{n_{2 j+1}}$-dependent. Condition (2) is achieved by establishing the following

Claim. Given $\mathcal{T} \in \mathfrak{D}$, there exist intervals $J_{1}<\ldots<J_{s}$ in $\{1, \ldots, p\}$ so that: 
(1) $\left\{z_{\min J_{t}}: t \leq s\right\}$ is $S_{f_{2 j+1}+1^{-}}$-admissible.

(2) $\mu_{\mathcal{T}} \mid\left\{z_{i}: i \in J_{t}\right\}$ is constant for all $t \leq s$.

(3) $\left|\mu_{\mathcal{T}}\left(z_{i}\right)\right|<14 / m_{2 j+1}^{2}$ for all $i \notin \bigcup_{t=1}^{s} J_{t}$.

To prove the claim suppose first that $w(\mathcal{T})>m_{2 j+1}$. Corollary 4.11 shows that $\left|\mu_{\mathcal{T}}\left(z_{i}\right)\right| \geq 14 / m_{2 j+1}^{2}$ for at most one $i \leq p$, and thus the claim holds in this case.

Next assume that $w(\mathcal{T})=m_{2 j+1}$. Without loss of generality, there exist an $S_{n_{2 j+1}}$-dependent sequence $\mathcal{R}_{1}<\ldots<\mathcal{R}_{l}$ in $\mathfrak{D}$ and an interval $J$ so that $\mu_{\mathcal{T}}=\left(1 / m_{2 j+1}\right) \sum_{k=1}^{l} \mu_{\mathcal{R}_{k}} \mid J$. Let $i_{0}$ be the largest $i$ for which $w\left(\mathcal{T}_{i}\right)$ is an element of $\left\{w\left(\mathcal{R}_{k}\right): k \leq l\right\}$, and let $i_{0}=0$ if no such $i$ exists. The injectivity of $\sigma$ and Corollary 4.11 imply that if $i_{0} \in\{0,1\}$, or if $w\left(\mathcal{T}_{i_{0}}\right)=w\left(\mathcal{R}_{1}\right)$, then $\left|\mu_{\mathcal{T}}\left(z_{i}\right)\right|<14 / m_{2 j+1}^{2}$ for all $i \neq i_{0}$.

If $i_{0}>1$ and $w\left(\mathcal{T}_{i_{0}}\right) \neq w\left(\mathcal{R}_{1}\right)$, then the injectivity of $\sigma$ yields $w\left(\mathcal{T}_{i_{0}}\right)=$ $w\left(\mathcal{R}_{i_{0}}\right)$ and $\mathcal{T}_{i}=\mathcal{R}_{i}$ for $i<i_{0}$. It now follows by Corollary 4.11 that $\left|\mu_{\mathcal{T}}\left(z_{i}\right)\right|<14 / m_{2 j+1}^{2}$ for all $i>i_{0}$. We also observe that there exists $i_{1}<i_{0}$ such that $\mu_{\mathcal{T}}\left(z_{i}\right)=0$ if $i<i_{1}$, while $\mu_{\mathcal{T}}\left(z_{i}\right)=1 / m_{2 j+1}$ if $i_{1}<i<i_{0}-1$. Concluding, there exist four intervals $J_{1}<J_{2}<J_{3}<J_{4}$ in $\{1, \ldots, p\}$, some possibly empty, such that $\mu_{\mathcal{T}} \mid\left\{z_{i}: i \in J_{t}\right\}$ is constant for every $t \leq 4$, while $\left|\mu_{\mathcal{T}}\left(z_{i}\right)\right|<14 / m_{2 j+1}^{2}$ for each $i \notin \bigcup_{t=1}^{4} J_{t}$.

Finally, assume $w(\mathcal{T})<m_{2 j+1}$. If $w(\mathcal{T})=1$, the claim trivially holds since in that case $\left|\mu_{\mathcal{T}}\left(z_{i}\right)\right|<14 / m_{2 j+1}^{2}$ for every $i \leq p$. So suppose that $w(\mathcal{T})>1$. Choose $\mathcal{G}_{0} \subset \mathfrak{D} S_{f_{2 j+1}}$-admissible and scalars $\left(\lambda_{\mathcal{R}}\right)_{\mathcal{R} \in \mathcal{G}_{0}}$ according to the decomposition Lemma 4.6. By splitting the $z_{i}$ 's into two sets, those whose support intersects at least two ranges of $\mu_{\mathcal{R}}$ 's, and those whose support intersects at most one, we deduce from our previous work and Remark 2.2 that there exist intervals $J_{1}<\ldots<J_{s}$ in $\{1, \ldots, p\}$ so that $\left\{z_{\min J_{t}}: t \leq s\right\}$ is $7 S_{f_{2 j+1}}$-admissible, $\mu_{\mathcal{T}} \mid\left\{z_{i}: i \in J_{t}\right\}$ is constant for all $t \leq s$, and $\left|\mu_{\mathcal{T}}\left(z_{i}\right)\right|<14 / m_{2 j+1}^{2}$ for all $i \notin \bigcup_{t=1}^{s} J_{t}$. Thus the claim holds and the proof is complete.

\section{References}

[1] D. Alspach and S. A. Argyros, Complexity of weakly null sequences, Dissertationes Math. 321 (1992).

[2] G. Androulakis and E. Odell, Distorting mixed Tsirelson spaces, Israel J. Math. 109 (1999), 125-149.

[3] S. A. Argyros, A universal property of reflexive hereditarily indecomposable Banach spaces, Proc. Amer. Math. Soc. 129 (2001), 3231-3238.

[4] S. A. Argyros and I. Deliyanni, Examples of asymptotic $\ell_{1}$ Banach spaces, Trans. Amer. Math. Soc. 349 (1997), 973-995.

[5] S. A. Argyros, I. Deliyanni, D. N. Kutzarova and A. Manoussakis, Modified mixed Tsirelson spaces, J. Funct. Anal. 159 (1998), 43-109. 
[6] S. A. Argyros and V. Felouzis, Interpolating hereditarily indecomposable Banach spaces, J. Amer. Math. Soc. 13 (2000), 243-294.

[7] S. A. Argyros and I. Gasparis, Unconditional structures of weakly null sequences, Trans. Amer. Math. Soc. 353 (2001), 2019-2058.

[8] S. A. Argyros, S. Mercourakis and A. Tsarpalias, Convex unconditionality and summability of weakly null sequences, Israel J. Math. 107 (1998), 157-193.

[9] P. G. Casazza and T. Shura, Tsirelson's Space, Lecture Notes in Math. 1363, Springer, 1989.

[10] E. E. Ellentuck, A new proof that analytic sets are Ramsey, J. Symbolic Logic 39 (1974), 163-165.

[11] T. Figiel and W. B. Johnson, A uniformly convex Banach space which contains no $\ell_{p}$, Compositio Math. 29 (1974), 179-190.

[12] I. Gasparis, A dichotomy theorem for subsets of the power set of the natural numbers, Proc. Amer. Math. Soc. 129 (2001), 759-764.

[13] I. Gasparis and D. H. Leung, On the complemented subspaces of the Schreier spaces, Studia Math. 141 (2000), 273-300.

[14] W. T. Gowers, A new dichotomy for Banach spaces, Geom. Funct. Anal. 6 (1996), 1083-1093.

[15] W. T. Gowers and B. Maurey, The unconditional basic sequence problem, J. Amer. Math. Soc. 6 (1993), 851-874.

[16] A. Kechris, Classical Descriptive Set Theory, Grad. Texts in Math. 156, Springer, Berlin, 1994.

[17] K. Kuratowski, Applications of the Baire-category method to the problem of independent sets, Fund. Math. 81 (1973), 65-72.

[18] J. Lindenstrauss and L. Tzafriri, Classical Banach Spaces I, Springer, New York, 1977.

[19] B. Maurey, V. D. Milman and N. Tomczak-Jaegermann, Asymptotic infinite-dimensional theory of Banach spaces, in: Oper. Theory: Adv. Appl. 77, Birkhäuser, 1994, 149-175.

[20] B. Maurey and H. P. Rosenthal, Normalized weakly null sequences with no unconditional subsequences, Studia Math. 61 (1977), 77-98.

[21] J. Mycielski, Almost every function is independent, Fund. Math. 81 (1973), 43-48.

[22] E. Odell, Applications of Ramsey theorems to Banach space theory, in: Notes in Banach Spaces, H. E. Lacey (ed.), Univ. Texas Press, 1980, 379-404.

[23] - On subspaces, asymptotic structure, and distortion of Banach spaces; connections with logic, in: Analysis and Logic, C. Finet and C. Michaux (eds.), 2000, 301-376 (to appear).

[24] E. Odell and T. Schlumprecht, The distortion problem, Acta Math. 173 (1994), 259-281.

[25] E. Odell and N. Tomczak-Jaegermann, On certain equivalent norms on Tsirelson's space, Illinois J. Math. 44 (2000), 51-71.

[26] E. Odell, N. Tomczak-Jaegermann and R. Wagner, Proximity to $\ell_{1}$ and distortion in asymptotic $\ell_{1}$ spaces, J. Funct. Anal. 150 (1997), 101-145.

[27] T. Schlumprecht, An arbitrarily distortable Banach space, Israel J. Math. 76 (1991), 81-95.

[28] J. Schreier, Ein Gegenbeispiel zur Theorie der schwachen Konvergenz, Studia Math. 2 (1930), 58-62.

[29] N. Tomczak-Jaegermann, Banach spaces of type $p$ have arbitrarily distortable subspaces, Geom. Funct. Anal. 6 (1996), 1074-1082. 
[30] B. S. Tsirelson, Not every Banach space contains $\ell_{p}$ or $c_{0}$, Funct. Anal. Appl. 8 (1974), 138-141.

Department of Mathematics

Current address:

Oklahoma State University

Stillwater, OK 74078-1058, U.S.A.

E-mail: ioagaspa@math.okstate.edu

Department of Mathematics

University of Crete

Knossou Avenue, P.O. Box 2208

Herakleion, Crete 71409, Greece

E-mail: ioagaspa@math.uch.gr

Received October 30, 2001

Revised version December 11, 2001 